\title{
Second-order equation of state with the Skyrme interaction: Cutoff and dimensional regularization with the inclusion of rearrangement terms
}

\author{
C. J. Yang, ${ }^{1}$ M. Grasso, ${ }^{1}$ X. Roca-Maza, ${ }^{2,3}$ G. Colò, ${ }^{2,3}$ and K. Moghrabi ${ }^{4,5}$ \\ ${ }^{1}$ Institut de Physique Nucléaire, CNRS-IN2P3, Université Paris-Sud, Université Paris-Saclay, 91406 Orsay, France \\ ${ }^{2}$ Dipartimento di Fisica, Università degli Studi di Milano, Via Celoria 16, 20133 Milano, Italy \\ ${ }^{3}$ INFN, Sezione di Milano, Via Celoria 16, 20133 Milano, Italy \\ ${ }^{4}$ Faculty of Sciences, Lebanese University, Beirut, Lebanon \\ ${ }^{5}$ American University of Science and Technology, Beirut, Lebanon
}

(Received 15 January 2016; revised manuscript received 25 April 2016; published 8 September 2016)

\begin{abstract}
We evaluate the second-order (beyond-mean-field) contribution to the equation of state of nuclear matter with the effective Skyrme force and use cutoff and dimensional regularizations to treat the ultraviolet divergence produced by the zero-range character of this interaction. An adjustment of the force parameters is then performed in both cases to remove any double counting generated by the explicit computation of beyond-mean-field corrections with the Skyrme force. In addition, we include at second order the rearrangement terms associated with the density-dependent part of the Skyrme force and discuss their effect. Sets of parameters are proposed to define new effective forces which are specially designed for second-order calculations in nuclear matter.
\end{abstract}

DOI: 10.1103/PhysRevC.94.034311

\section{INTRODUCTION}

The energy-density-functional (EDF) theory has been developed in nuclear physics over the last several decades. In this theoretical framework, the energy of the system is computed by using functionals of the density, which are usually derived from effective interactions [1], with some exceptions (see, for instance, the work reported in Ref. [2]). Mean-field-type models constitute the basis on which this theory was constructed starting from the 1970s. Such models have strong analogies with the leading order of the many-body Dyson perturbative expansion [3], based on the independentparticle approximation, and are currently applied to numerous many-particle systems.

Mean-field-based models are extensively employed in the study of medium-mass and heavy nuclei, and are particularly successful in describing with a good accuracy a large number of known masses and radii. Despite this success, more sophisticated beyond-mean-field models are necessary, for instance, to perform accurate spectroscopic analyses for nuclear ground states or to provide reliable descriptions of the physical fragmentation of nuclear excitations. In these cases, additional correlations, with respect to what is contained in a mean-field picture, have to be included in the theoretical scheme. Going beyond the mean field, with respect to the many-body Dyson perturbative expansion, implies including higher orders. This is a challenging task to accomplish for several reasons: among them, the highly increased numerical cost, and the conceptual problems implied by the choice of the interaction to be used. Some of these problems are mentioned in what follows. Within the EDF theory, the currently used density functionals are usually produced by phenomenological interactions or Lagrangians in the nonrelativistic and relativistic cases, respectively. Skyrme [4,5] and Gogny [6,7] forces are the most used interactions in the nonrelativistic framework. The fitting procedure of their parameters is performed with mean-field calculations for nuclear matter and some chosen nuclei. It is obvious that, if such interactions are used in cases where higher orders are explicitly included in the theoretical models, double-counting problems arise. Furthermore, in those cases where the effective interactions have a zero range, ultraviolet divergences may occur beyond the mean field and have to be treated.

Our objective is to construct a generalized EDF framework, where new effective interactions are introduced, which are now designed to treat both matter and finite nuclei in beyondmean-field models, avoiding double-counting problems and regularizing ultraviolet divergences. We started this work with the Skyrme interaction [8-10]. Second-order calculations were performed to compute the equation of state (EOS) of symmetric, neutron, and asymmetric matter. First, only symmetric matter and a simplified form of the Skyrme interaction (contact interaction with a density-dependent coupling constant) were analyzed [8]. This simplified Skyrme model corresponds to the so-called $t_{0}-t_{3}$ model, where the velocity-dependent, the spin-orbit, and the tensor terms of the Skyrme force are omitted. The second-order contribution was evaluated analytically and its cutoff-dependent part was identified. The divergent part was found to have a linear asymptotic behavior with respect to a momentum cutoff. The set of parameters (three in that case) were adjusted on a benchmark EOS for several values of the cutoff. These parameter sets were recently employed in a simplified test-calculation for the nucleus ${ }^{16} \mathrm{O}$ [11]. The second-order correction to the total binding energy was evaluated in this nucleus and encouraging results were found, indicating a reasonable convergence with respect to the chosen cutoff.

We then performed the same type of procedure (analytical derivation of the second-order contribution and adjustment of the parameters) by including also the velocity-dependent terms in the Skyrme interaction. Two directions were explored, namely (i) keeping the cutoff-dependent terms [9] and (ii) applying the dimensional regularization technique to extract only the finite part [10]. 
We recently realized that some aspects of the formal derivation on which both studies $[9,10]$ were based are not correct, and we aim here at providing the correct full formulas and results. For the sake of clarity and to present a self-contained reference on the topic, we provide here all the needed details via reference to previous literature, new formulas and, for an easy comparison with Refs. [9,10], the same type of figures. In addition, we illustrate the effect on the EOS related to the inclusion of the proper rearrangement terms at second order. Rearrangement terms were neglected in Refs. $[9,10]$. The general way of computing them in beyond-mean-field models was discussed in Ref. [12].

The ultraviolet divergence at second order is handled in two ways: (i) by using a cutoff regularization, as in Ref. [9]. The cutoff-dependent results are adjusted on a benchmark EOS and several sets of parameters are generated for each chosen value of the cutoff; (ii) by applying the dimensional-regularization procedure. Only the finite parts of the second-order corrections are thus extracted as in Ref. [10] and the cutoff-dependent part is eliminated by the dimensional regularization. A unique set of parameters is thus produced by adjusting on a benchmark EOS.

As a first approximation, in the first part of the article we replace the effective mass $m^{*}$ with the bare mass $m$, as done in Ref. [13] (this approximation will be abandoned in the last part of the article). We find in this case that the finite part of the EOS calculated for symmetric and neutron matter is consistent with the recent results shown in Ref. [13]. There are differences from Ref. [13]: (1) The tensor and spin-orbit parts are omitted for simplicity in the interaction. For the tensor part, this is first justified by the fact that many currently used Skyrme interactions do not contain such terms. Spin-orbit and tensor terms do not contribute to the first-order EOS. In principle, they contribute when the second order is included. ${ }^{1}$ (2) We also calculate the cutoff-dependent part in the secondorder contribution because we perform a cutoff regularization. (3) Also asymmetric matter is treated here. (4) The densitydependent part of the Skyrme interaction is not omitted. It is well known that this term is necessary to reproduce the correct saturation point of symmetric matter at first order. In Ref. [13] it is stressed that this term is indeed necessary for properly reproducing the saturation density of symmetric matter also at second order. The second-order curves obtained there by omitting the density-dependent term do not reproduce at all the saturation point, providing a saturation density of $\sim 0.22 \mathrm{fm}^{-3}$. It is well known that the inclusion of such a term may be problematic in many respects (see, for instance, Ref. [14]). Several drawbacks related to the density dependence were identified, such as the existence of pathologies in some

\footnotetext{
${ }^{1}$ We note that such terms were taken into account in Ref. [13]; however, the adjustment of the parameters performed in that work for the second-order EOS of symmetric matter (done by omitting the density-dependent term) provided $W_{0}=t_{4}=t_{5}=0$, where $W_{0}, t_{4}$, and $t_{5}$ are the parameters that tune the spin-orbit $\left(W_{0}\right)$ and tensor $\left(t_{4}\right.$ and $t_{5}$ ) terms introduced in Ref. [13].
}

applications of the generator-coordinate method [15]. Our pragmatic choice is to use density-dependent interactions as a starting point in our work because of their good performance, and we leave for a future work the discussion of the associated problems.

Note that, despite the explicit density dependence in the interaction, the Hugenholtz-van Howe theorem [16] is satisfied in the Skyrme case whenever the proper rearrangement terms (associated with the density dependence) are explicitly introduced, as is usually done within the random-phase approximation (RPA) and as was recently done also in the specific case of the second RPA [17]. Reference [18] pointed out that the matrix elements of the interaction used for the computation of the energy in second-order perturbation theory are related to $B$ RPA matrix elements. The RPA residual interaction (second derivative of the Hartree-Fock energy functional [19], which automatically includes the rearrangement terms) has thus to be used to calculate the second-order energy correction. As a first approximation, as done in Refs. [9,10], we neglect rearrangement terms. We then include them (together with the effective mass) in the last part of the article for symmetric and neutron matter and provide sets of parameters where their effect is taken into account. Such sets of parameters may be considered a very reasonable starting point to construct a bridge between infinite matter, where our effective regularized interactions are presently adjusted, and finite nuclei, where we eventually plan to employ them.

We analyze here the second-order integrals using two choices for the momentum cutoff. While the numerical Monte Carlo integration is performed with a cutoff $(\Lambda)$ on the transferred momentum $\mathbf{q}$, it turns out that the use of a cutoff $(\lambda)$ on the outgoing relative momentum $\mathbf{k}^{\prime}$ is more convenient for the analytical derivation. This choice is analogous to that adopted for instance in the low-momentum interaction $V_{\text {low- } k}$ [20]. The analytical derivation is performed here only for symmetric and pure neutron matter. For asymmetric matter, we solve numerically the second-order integrals with the Monte Carlo method. To present a coherent analysis of the obtained results and discuss figures with a unique choice for the momentum cutoff, we show in this article, in the case of the cutoff regularization, second-order EOS's obtained in all cases numerically, with a cutoff equal to $\Lambda$.

The article is organized as follows. In Sec. II the cutoff regularization is discussed. First, the analytical expressions of the second-order EOS's are shown in the cases of symmetric and pure neutron matter (Sec. II A). Numerical results for the second-order EOS's, obtained with the Monte Carlo method, are then shown for several values of the cutoff $\Lambda$ for symmetric, neutron, and asymmetric matter. Adjustments of parameters are presented and discussed (Sec. II B). In Sec. III the dimensional-regularized results are illustrated and the adjustment of the parameters is discussed also in this case. Section IV illustrates results obtained for symmetric and neutron matter in the case where the approximation $m^{*}=m$ is not employed ( $m^{*}$ is taken equal to its mean-field value) and rearrangement terms are included. Sets of parameters are provided. We draw conclusions in Sec. V. The Appendix lists the factors appearing in front of the different types of integrals which are solved by the Monte Carlo method. 


\section{CUTOFF REGULARIZATION}

A. Analytical derivation of the second-order contribution for symmetric and neutron matter

We start by writing the standard Skyrme interaction,

$$
\begin{aligned}
v\left(\mathbf{k}, \mathbf{k}^{\prime}\right)= & t_{0}\left(1+x_{0} P_{\sigma}\right)+\frac{1}{2} t_{1}\left(1+x_{1} P_{\sigma}\right)\left(\mathbf{k}^{\prime 2}+\mathbf{k}^{2}\right) \\
& +t_{2}\left(1+x_{2} P_{\sigma}\right) \mathbf{k}^{\prime} \cdot \mathbf{k}+\frac{1}{6} t_{3}\left(1+x_{3} P_{\sigma}\right) \rho^{\alpha},
\end{aligned}
$$

where we adopt the following convention,

$$
v\left(\mathbf{k}, \mathbf{k}^{\prime}\right)=\iint d^{3} \mathbf{r} d^{3} \mathbf{r}^{\prime} e^{-i \mathbf{k} \cdot \mathbf{r}} v\left(\mathbf{r}, \mathbf{r}^{\prime}\right) e^{i \mathbf{k}^{\prime} \cdot \mathbf{r}^{\prime}}
$$

As already anticipated, we have omitted the spin-orbit and tensor parts, for simplicity. The parameters $\left(t_{i}, x_{i}\right.$, and $\alpha$ ) are in this case nine. $P_{\sigma}$ is the spin-exchange operator, $P_{\sigma}=$ $\frac{1}{2}\left(1+\sigma_{1} \cdot \sigma_{2}\right)$. The mean-field or first-order contribution to the EOS of symmetric matter is the well-known expression

$$
\begin{aligned}
\frac{E_{\mathrm{sym}}^{(1)}}{A}= & \frac{3}{10} \frac{\hbar^{2}}{m}\left(\frac{3 \pi^{2}}{2}\right)^{\frac{2}{3}} \rho^{\frac{2}{3}}+\frac{3}{8} t_{0} \rho+\frac{3}{80}\left(\frac{3 \pi^{2}}{2}\right)^{\frac{2}{3}} \Theta_{S} \rho^{\frac{5}{3}} \\
& +\frac{1}{16} t_{3} \rho^{\alpha+1},
\end{aligned}
$$

where $\Theta_{S}=3 t_{1}+t_{2}\left(5+4 x_{2}\right)$. For neutron matter, the firstorder contribution to the EOS is written as

$$
\begin{aligned}
\frac{E_{\text {neutr }}^{(1)}}{A}= & \frac{3}{10} \frac{\hbar^{2}}{m}\left(3 \pi^{2}\right)^{\frac{2}{3}} \rho^{\frac{2}{3}}+\frac{1}{4} t_{0} \rho\left(1-x_{0}\right) \\
& +\frac{3}{40}\left(3 \pi^{2}\right)^{\frac{2}{3}}\left(\Theta_{S}-\Theta_{V}\right) \rho^{\frac{5}{3}}+\frac{1}{24} t_{3} \rho^{\alpha+1}\left(1-x_{3}\right),
\end{aligned}
$$

with $\Theta_{V}=t_{1}\left(2+x_{1}\right)+t_{2}\left(2+x_{2}\right)$. In symmetric matter, the total density and the Fermi momentum $k_{F}$ are related by the relation $k_{F}=\left(\frac{3 \pi^{2}}{2} \rho\right)^{1 / 3}$. Neutron and proton Fermi momenta are in this case equal to $k_{F}$. In neutron matter, the Fermi

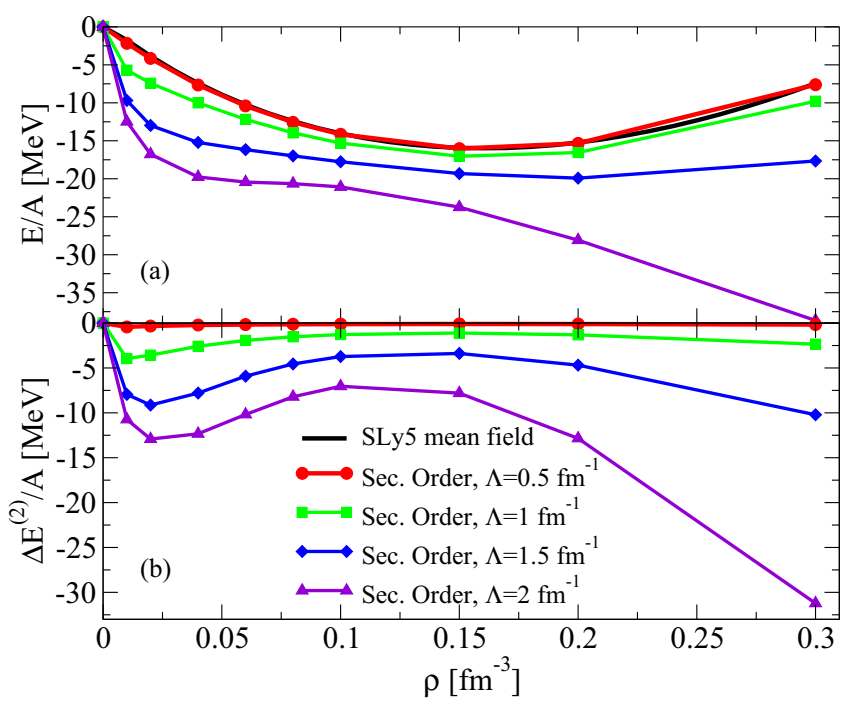

FIG. 1. (a) Second-order EOS of symmetric matter computed for several values of the cutoff $\Lambda$ and compared with the mean-field EOS; (b) second-order correction to the energy per particle for symmetric matter. The used parameters are those of SLy5.

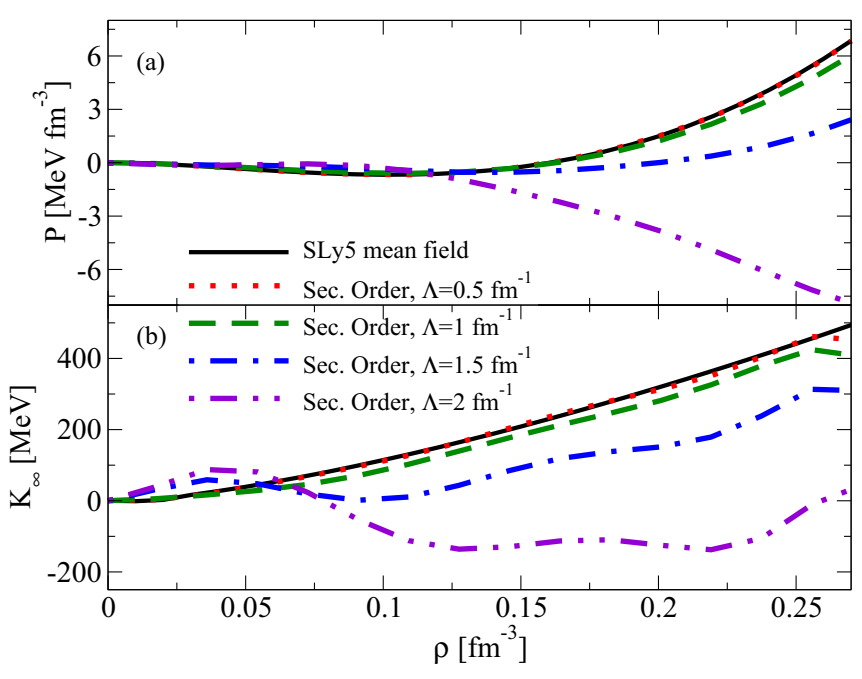

FIG. 2. (a) Second-order pressure; (b) second-order incompressibility modulus. The used parameters are those of SLy5.

momentum $k_{F_{N}}$ is related to the total density (which is equal to the neutron density) by the relation $k_{F_{N}}=\left(3 \pi^{2} \rho\right)^{1 / 3}$.

The second-order contribution is calculated by solving the integral

$$
\Delta E^{(2)}=-\frac{1}{4} \frac{\Omega^{3}}{(2 \pi)^{9}} \int d^{3} \mathbf{k}_{\mathbf{1}} \int d^{3} \mathbf{k}_{\mathbf{2}} \int d^{3} \mathbf{q} \frac{\left|\left\langle\mathbf{k}_{\mathbf{1}} \mathbf{k}_{\mathbf{2}}|V| \mathbf{k}_{\mathbf{1}}^{\prime} \mathbf{k}_{\mathbf{2}}^{\prime}\right\rangle\right|^{2}}{\epsilon_{1}^{\prime}+\epsilon_{2}^{\prime}-\epsilon_{1}-\epsilon_{2}}
$$

where $V=v / \Omega, \Omega$ is the box volume where the wave functions are normalized, $v$ is the Skyrme interaction written in Eq. (1), $\mathbf{k}_{\mathbf{1}}^{\prime}=\mathbf{q}+\mathbf{k}_{\mathbf{1}}, \mathbf{k}_{\mathbf{2}}^{\prime}=\mathbf{k}_{\mathbf{2}}-\mathbf{q}$, and $\mathbf{q}$ is the transferred momentum. Eq. (3) may be written as

$$
\Delta E^{(2)}=\frac{1}{4} \frac{\Omega}{(2 \pi)^{9}} \int d^{3} \mathbf{k}_{\mathbf{1}} \int d^{3} \mathbf{k}_{\mathbf{2}} \int d^{3} \mathbf{q}\left|\left\langle\mathbf{k}_{\mathbf{1}} \mathbf{k}_{\mathbf{2}}|v| \mathbf{k}_{\mathbf{1}}^{\prime} \mathbf{k}_{\mathbf{2}}^{\prime}\right\rangle\right|^{2} G,
$$

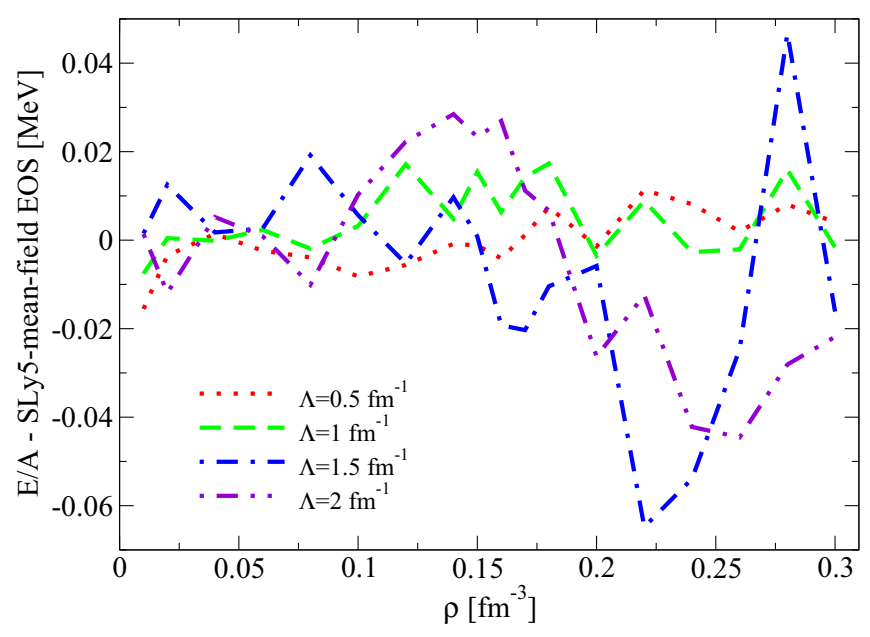

FIG. 3. Difference between the refitted second-order EOS and the mean-field EOS for symmetric matter. 


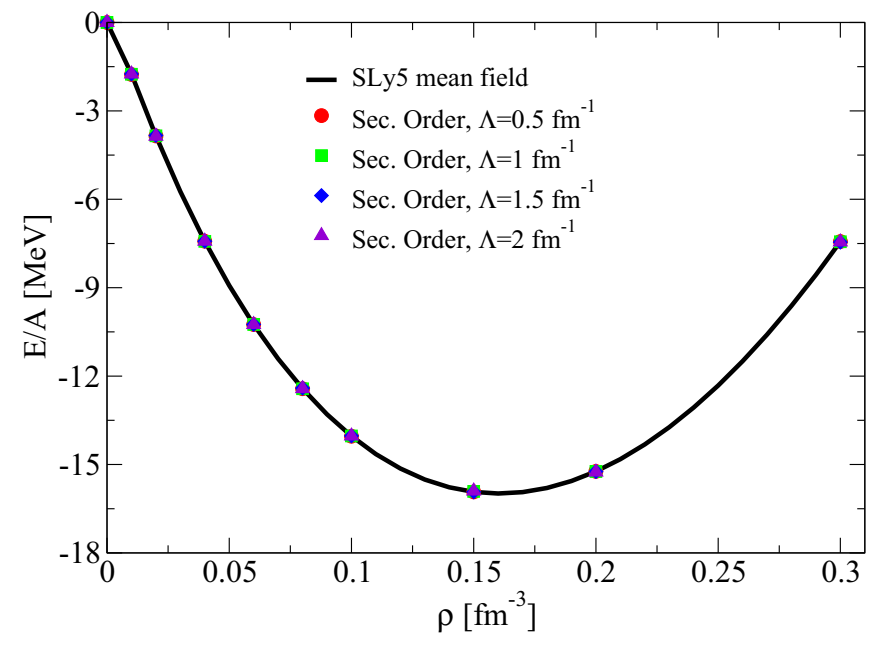

FIG. 4. Second-order refitted EOS compared with the SLy5mean-field EOS for symmetric matter.

where we have introduced the propagator $G$,

$$
G=\frac{-1}{\epsilon_{1}^{\prime}+\epsilon_{2}^{\prime}-\epsilon_{1}-\epsilon_{2}}, \quad \epsilon_{i}^{(\prime)}=\frac{\hbar^{2} k_{i}^{(\prime) 2}}{2 m_{i}^{*}},
$$

with

$$
\begin{aligned}
\left|\mathbf{k}_{\mathbf{1}}\right|<k_{F 1}, & & \left|\mathbf{k}_{\mathbf{2}}\right|<k_{F 2}, \\
\left|\mathbf{q}+\mathbf{k}_{\mathbf{1}}\right|>k_{F 1}, & & \left|\mathbf{k}_{\mathbf{2}}-\mathbf{q}\right|>k_{F 2} .
\end{aligned}
$$

In Eq. (5), $m_{i}^{*}$ represents the nucleonic effective mass. In this work, we first take the approximation $m_{i}^{*}=m$ for simplicity. Such approximation will not be adopted in Sec. IV. As is well known, the $k$ dependence of the potential $U$ is absorbed in the effective mass while a constant term in $U$ cancels out in the denominator of Eq. (3) and is consequently not written.

In the integral of Eq. (4), $\mathbf{k}_{\mathbf{1}}$ and $\mathbf{k}_{\mathbf{2}}$ lie inside the Fermi spheres associated with $k_{F 1}$ and $k_{F 2}$, respectively, and the integrals on $\mathbf{k}_{\mathbf{1}}$ and $\mathbf{k}_{\mathbf{2}}$ do not diverge. An ultraviolet divergence appears in the computation of the integral on the transferred momentum $\mathbf{q}$ and a cutoff $\Lambda$ is put in that integral as a regulator.

We now introduce the incoming $\mathbf{k}$ and outgoing $\mathbf{k}^{\prime}$ relative momenta, appearing in the Skyrme interaction, Eq. (1), and

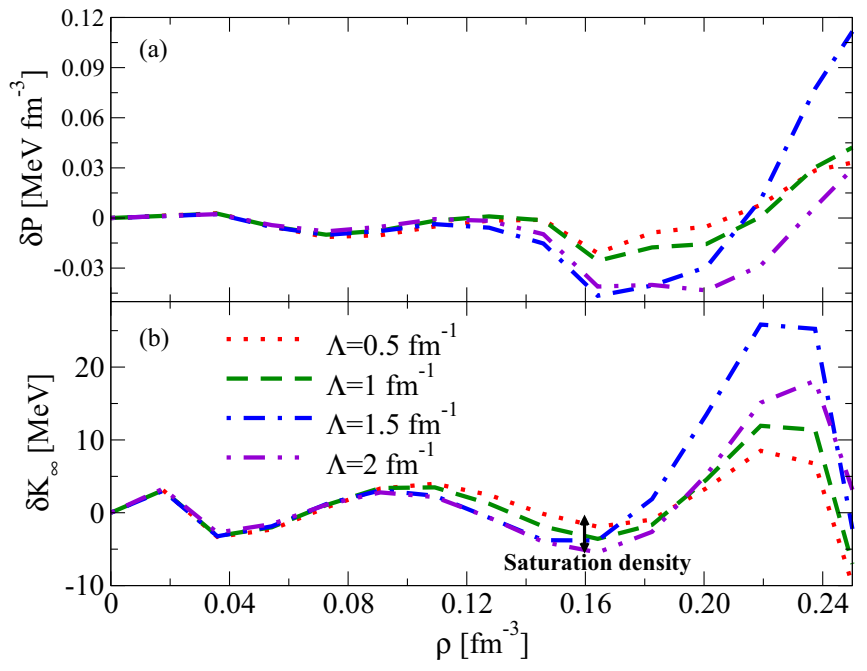

FIG. 5. (a) Difference between the second-order pressure and the mean-field-SLy5 value; (b) same as in panel (a), but for the incompressibility modulus. The black arrow indicates the maximum deviation of the compressibility from the mean-field-SLy5 value at the saturation point.

related to $\mathbf{k}_{\mathbf{1}}, \mathbf{k}_{\mathbf{2}}$, and $\mathbf{q}$ by

$$
\mathbf{k}=\frac{\mathbf{k}_{\mathbf{1}}-\mathbf{k}_{\mathbf{2}}}{2}, \quad \mathbf{k}^{\prime}=\frac{\mathbf{k}_{\mathbf{1}}^{\prime}-\mathbf{k}_{\mathbf{2}}^{\prime}}{2}=\frac{\mathbf{k}_{\mathbf{1}}-\mathbf{k}_{\mathbf{2}}}{2}+\mathbf{q}
$$

In this work, the analytical derivation of the second-order contribution to the EOS has been done following Ref. [21]. This specific derivation can be adapted only to the cases of symmetric and pure neutron matter, where there is a unique Fermi momentum $k_{F 1}=k_{F 2}$, and not to the case of asymmetric matter, where $k_{F 1} \neq k_{F 2}$. For asymmetric matter, the EOS is computed numerically by a Monte Carlo integration and discussed in Sec. II B together with all the other numerical results. In the present section, as well as in the following, we neglect the rearrangement terms associated to the densitydependent part of the interaction. Such terms will be included in Sec. IV.

\section{Symmetric matter}

In symmetric matter as well as in neutron matter (next subsection) it is advantageous to perform the change of

TABLE I. Parameter sets obtained with the fit of the second-order EOS of symmetric matter for different values of the cutoff $\Lambda$ compared with the original set SLy5. In the last column, the $\chi^{2}$ values are shown.

\begin{tabular}{lcccccccccc}
\hline \hline & $\begin{array}{c}t_{0} \\
\left(\mathrm{MeV} \mathrm{fm}^{3}\right)\end{array}$ & $\begin{array}{c}t_{1} \\
\left(\mathrm{MeV} \mathrm{fm}^{5}\right)\end{array}$ & $\begin{array}{c}t_{2} \\
\left(\mathrm{MeV} \mathrm{fm}^{5}\right)\end{array}$ & $\begin{array}{c}t_{3} \\
\left(\mathrm{MeV} \mathrm{fm}^{3+3 \alpha}\right)\end{array}$ & $x_{0}$ & $x_{1}$ & $x_{2}$ & $x_{3}$ & $\alpha$ & $\chi^{2}$ \\
\hline SLy5 & -2484.88 & 483.13 & -549.40 & 13763.0 & 0.778 & -0.328 & -1.0 & 1.267 & 0.16667 & \\
$\Lambda\left(\mathrm{fm}^{-1}\right)$ & & & & & & & & & & \\
0.5 & -1461.868 & 497.986 & -1471.462 & 9915.064 & 0.5360 & -1.529 & -1.068 & 8.298 & 0.3201 & $2.08 \times 10^{-3}$ \\
1.0 & -1207.550 & 645.148 & -1361.666 & 6942.115 & 0.4854 & -2.106 & -1.092 & 5.575 & 0.2831 & $1.46 \times 10^{-3}$ \\
1.5 & -1124.277 & 614.238 & -1063.666 & 5711.048 & 0.4333 & -2.690 & -1.349 & 5.103 & 0.2070 & $2.26 \times 10^{-2}$ \\
2.0 & -530.285 & 227.301 & -389.370 & 8927.183 & 0.9383 & -0.7346 & -0.3959 & 0.4783 & 0.6680 & $3.13 \times 10^{-2}$ \\
\hline \hline
\end{tabular}




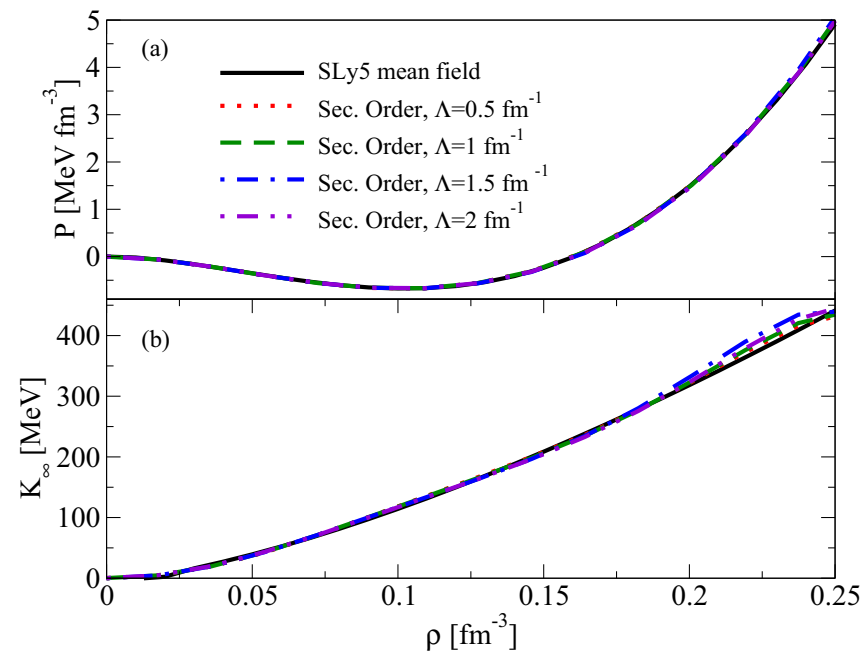

FIG. 6. (a) Second-order pressure compared to the SLy5-meanfield curve; (b) same as in panel (a), but for the incompressibility modulus.

variables given by Eq. (7) to write the propagator as

$$
G=\frac{-m}{\hbar^{2}\left(k^{\prime 2}-k^{2}\right)} \text {. }
$$

Starting from Eq. (4), dividing by the number of particles $A=\Omega \rho$, writing explicitly the sums over spin and isospin and the direct and exchange terms, the second-order correction to the EOS is equal to

$$
\begin{aligned}
\frac{E_{\mathrm{sym}}^{(2)}}{A}= & -\frac{3 m}{32(2 \pi)^{7} \hbar^{2} k_{F}^{3}} \sum_{S T M_{S} M_{S^{\prime}}}(2 T+1) \iiint d^{3} \mathbf{K} d^{3} \mathbf{k} d^{3} \mathbf{k}^{\prime} \\
& \times \frac{\mid\left\langle\left. X_{M_{S}}^{S}\left|v_{S T}\left(\mathbf{k}, \mathbf{k}^{\prime}\right)-(-)^{S+T} v_{S T}\left(\mathbf{k},-\mathbf{k}^{\prime}\right)\right| X_{M_{S^{\prime}}}^{S}\right|^{2}\right.}{\left(k^{\prime 2}-k^{2}\right)} .
\end{aligned}
$$

Here $S$ and $T$ are the total spin and isospin, respectively, $M_{S^{(\prime)}}$ is the projection of $S$ on the $z$ axis, and $X_{M_{S^{(\prime)}}}^{S}$ is the two-body spin state. The interaction $v_{S T}$ is always the interaction $v$ of Eq. (1), after having evaluated the expectation value in the isospin state, and where we have explicitly indicated spin and isospin labels for convenience. Note that the additional factor $1 /(2 \pi)^{6}$ in Eq. (9), with respect to the corresponding expression in Ref. [21], comes from the different convention adopted in Eq. (2). The two terms in Eq. (9) represent the direct and

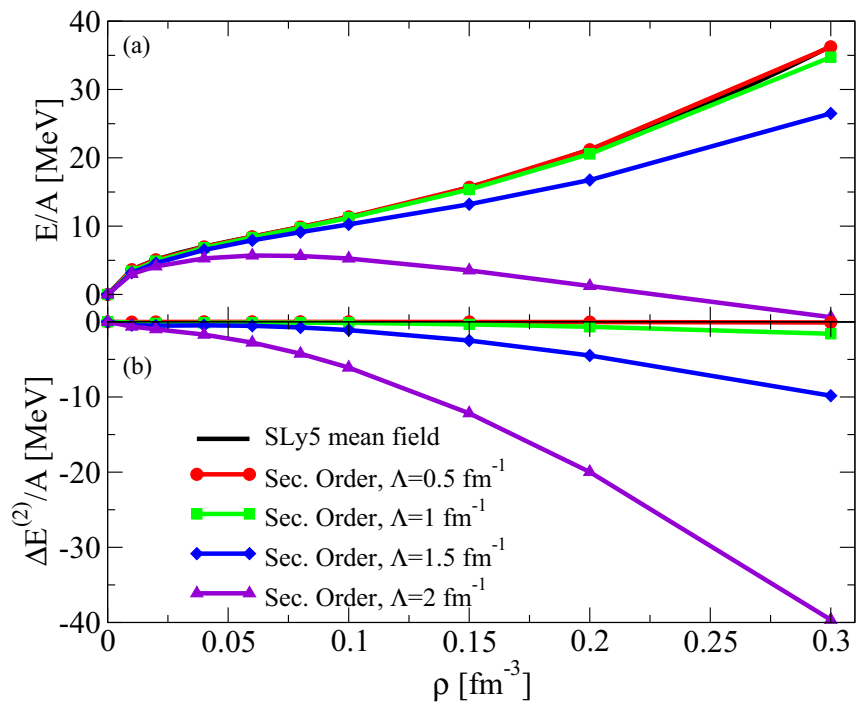

FIG. 7. (a) Second-order EOS of neutron matter for several values of the cutoff, calculated with the SLy5 parameters, and SLy5-meanfield EOS; (b) second-order correction.

exchange contributions. We have introduced a third variable $\mathbf{K}$, which is chosen in the same way as in Ref. [21]; that is, $\mathbf{K} \equiv \mathbf{k}_{1}+\mathbf{k}_{2}$. Since both $\mathbf{k}_{\mathbf{1}}$ and $\mathbf{k}_{\mathbf{2}}$ lie inside the Fermi sphere associated with $k_{F}$, the integrals on the incoming relative momentum $\mathbf{k}$ and on $\mathbf{K}$ do not diverge. A regulator must however be put on the diverging integral in $\mathbf{k}^{\prime}$ (we call this cutoff $\tilde{\lambda}$ and we will use later the dimensionless cutoff $\lambda=\tilde{\lambda} / k_{F}$ ). If this cutoff is chosen smaller than $k_{F}$, also the integral on the incoming momentum must be regulated by the same cutoff $\tilde{\lambda}$.

The interaction can be expanded in partial waves,

$$
v_{S T}\left(\mathbf{k}, \mathbf{k}^{\prime}\right)=\sum_{J M_{J} l l^{\prime}} v_{S T, l l^{\prime}}^{J}\left(k, k^{\prime}\right) y_{l S}^{J M_{J}}(\widehat{\mathbf{k}})\left[y_{l^{\prime} S}^{J M_{J}}\left(\widehat{\mathbf{k}}^{\prime}\right)\right]^{\dagger},
$$

where $y_{l S}^{J M_{J}}$ is written as

$$
y_{l S}^{J M_{J}}(\widehat{\mathbf{k}})=\sum_{m_{l}, M_{S}}\left\langle J M_{J} \mid l S m_{l} M_{S}\right\rangle Y_{l m_{l}}(\widehat{\mathbf{k}}) X_{M_{S}}^{S} .
$$

In general, $l$ and $l^{\prime}$ in Eq. (10) must have the same parity. By

\begin{tabular}{|c|c|c|c|c|c|c|c|c|c|c|}
\hline $\begin{array}{l}\text { SLy5 } \\
\Lambda\left(\mathrm{fm}^{-1}\right)\end{array}$ & -2484.88 & 483.13 & -549.40 & 13736.0 & 0.778 & -0.328 & -1.0 & 1.267 & 0.16667 & \\
\hline 0.5 & -1859.971 & 474.575 & -715.911 & 16306.843 & 0.7102 & -0.7273 & -0.9174 & 1.230 & 0.1625 & $2.19 \times 10^{-2}$ \\
\hline 1.0 & -3524.393 & 569.934 & -686.914 & 11329.194 & 1.048 & -0.7025 & -0.8657 & 1.680 & 0.08796 & $2.14 \times 10^{-2}$ \\
\hline 1.5 & -3924.552 & 677.302 & -724.123 & 11822.333 & 1.398 & -0.5270 & -0.9360 & 2.141 & 0.09518 & $3.01 \times 10^{-2}$ \\
\hline
\end{tabular}
imposing antisymmetrization, it holds that

$$
(-1)^{l+S+T}=(-1)^{l^{\prime}+S+T}=-1 .
$$

TABLE II. Parameter sets obtained with the fit of the second-order EOS of neutron matter for different values of the cutoff $\Lambda$ compared with the original set SLy5. The $\chi^{2}$ values are shown in the last column. 
This implies that the exchange term in Eq. (9) is equal to the direct term. After some manipulations, and, by evaluating the spin matrix elements, Eq. (9) becomes

$$
\begin{aligned}
\frac{E_{\text {sym }}^{(2)}}{A}= & -\frac{3 m}{16(2 \pi)^{8} \hbar^{2} k_{F}^{3}} \sum_{S T J \bar{J} L M l l^{\prime} \bar{l}^{\prime}} \delta_{S T l} \delta_{S T \bar{l}}(2 T+1)(2 J+1)(2 \bar{J}+1)(2 L+1)^{-1} \\
& \times\left[(2 l+1)\left(2 l^{\prime}+1\right)(2 \bar{l}+1)\left(2 \bar{l}^{\prime}+1\right)\right]^{1 / 2}\langle L 0 \mid l \bar{l} 00\rangle\left\langle L 0 \mid l^{\prime} \bar{l}^{\prime} 00\right\rangle W(J \bar{J} l \bar{l} ; L S) W\left(J \bar{J} l^{\prime} \bar{l}^{\prime} ; L S\right) \\
& \times \iiint d^{3} \mathbf{K} d^{3} \mathbf{k} d^{3} \mathbf{k}^{\prime} Y_{L M}(\hat{k}) Y_{L M}^{*}\left(\hat{k}^{\prime}\right) v_{S T, l l^{\prime}}^{J}\left(k, k^{\prime}\right) v_{S T, \bar{l} \bar{l}^{\prime}}^{\bar{J}}\left(k, k^{\prime}\right)\left(k^{\prime 2}-k^{2}\right)^{-1},
\end{aligned}
$$

with

$$
\delta_{S T l}=\frac{1}{2}\left[1-(-1)^{S+T+l}\right] .
$$

In Eq. (12), $W$ indicates Racah coefficients. In our case, the interaction is diagonal in $l$ and independent of $J$; that is, $v_{S T, l l^{\prime}}^{J}\left(k, k^{\prime}\right)=\delta_{l l^{\prime}} v_{S, T, l}\left(k, k^{\prime}\right)$. The product $\delta_{S T l} \delta_{S T l}$ in Eq. (12) implies that $l$ and $\bar{l}$ must have the same parity. This means that, for a given $(S, T)$, only even-even and odd-odd partial waves of the interaction may mix at second order. The Skyrme interaction of Eq. (1) contains only one type of even waves, the $s$-wave $t_{0}, t_{3}$, and $t_{1}$ terms, and one type of odd waves, the $p$-wave $t_{2}$ term. Consequently, $l$ and $\bar{l}$ must be the same (equal to 0 or 1 ) and the only quadratic terms that enter in the second-order contribution are proportional to $t_{0}^{2}, t_{3}^{2}, t_{1}^{2}, t_{0} t_{3}$, $t_{0} t_{1}, t_{3} t_{1}$, and $t_{2}^{2}$ (the only possible values for $L$ are $L=0$ and 2 for the $p$-wave case and $L=0$ for the $s$-wave case). Interference terms proportional to $t_{0} t_{2}, t_{3} t_{2}$, and $t_{1} t_{2}$ are absent in the EOS of symmetric and pure neutron matter. We stress that, on the other side, such interference terms are present in the EOS of asymmetric matter. This occurs due to the different Fermi momenta between neutrons and protons in asymmetric matter. Also the different effective masses of neutrons and protons would be responsible for such interference terms.

We write now explicitly the squares of the interaction $v_{S, T, l}^{2}\left(k, k^{\prime}\right)$ in the different channels. For the isovector case $T \stackrel{1}{=}$, one has $S=0\left(P_{\sigma}=-1\right)$ for $l=0$, and $S=1$ $\left(P_{\sigma}=1\right)$ for $l=1$. The square of the interaction is then written as

$$
\begin{array}{rl}
v_{S=0,}^{2} & T=1, l=0 \\
= & (4 \pi)^{2}\left[t_{0}^{2}\left(1-x_{0}\right)^{2}+\frac{1}{4} t_{1}^{2}\left(1-x_{1}\right)^{2}\left(k^{\prime 4}+2 k^{\prime 2} k^{2}+k^{4}\right)\right. \\
& +\frac{1}{36} t_{3}^{2}\left(1-x_{3}\right)^{2} \rho^{2 \alpha}+t_{0}\left(1-x_{0}\right) t_{1}\left(1-x_{1}\right)\left(k^{\prime 2}+k^{2}\right) \\
& +\frac{1}{6} t_{1}\left(1-x_{1}\right)\left(k^{\prime 2}+k^{2}\right) t_{3}\left(1-x_{3}\right) \rho^{\alpha} \\
& \left.+\frac{1}{3} t_{0}\left(1-x_{0}\right) t_{3}\left(1-x_{3}\right) \rho^{\alpha}\right]
\end{array}
$$

and

$$
v_{S=1, T=1, l=1}^{2}\left(k, k^{\prime}\right)=\frac{(4 \pi)^{2}}{9} t_{2}^{2}\left(1+x_{2}\right)^{2}\left(k^{\prime} k\right)^{2},
$$

for the two cases, respectively. Note that the factors $(4 \pi)^{2}$ and $(4 \pi)^{2} / 9$ in Eqs. (14) and (15), respectively, come from the partial wave expansion, Eq. (10), of the Skyrme interaction. In the isoscalar case $T=0$, one has $S=1\left(P_{\sigma}=1\right)$ for $l=0$ and $S=0\left(P_{\sigma}=-1\right)$ for $l=1$. The expressions for the square of the interaction $v_{S=1, T=0, l=0}^{2}\left(k, k^{\prime}\right)$ and $v_{S=0, T=0, l=1}^{2}\left(k, k^{\prime}\right)$ may be obtained from Eqs. (14) and (15), respectively, by substituting $\left(1+x_{i}\right)$ to $\left(1-x_{i}\right)$ in Eq. $(14)$ and $\left(1-x_{2}\right)$ to $\left(1+x_{2}\right)$ in Eq. (15).

We perform a change of variables to use dimensionless vectors,

$$
\mathbf{y}=\frac{\mathbf{k}}{k_{F}}, \quad \mathbf{y}^{\prime}=\frac{\mathbf{k}^{\prime}}{k_{F}}, \quad \mathbf{x}=\frac{\mathbf{K}}{2 k_{F}} .
$$

The new variables $\mathbf{y}$ and $\mathbf{y}^{\prime}$ should satisfy the conditions

$$
|\mathbf{y}|<1, \quad\left|\mathbf{y}^{\prime}\right|<\lambda .
$$

We integrate over all angles by using the function

$$
J_{L M}\left(x, y, y^{\prime}\right)=\iiint d \hat{x} d \hat{y} d \hat{y}^{\prime} Y_{L M}(\hat{y}) Y_{L M}^{*}\left(\hat{y}^{\prime}\right) .
$$

After some manipulations, one can write

$$
J_{L M}\left(x, y, y^{\prime}\right)=16 \pi^{2} \delta_{M, 0}(2 L+1) A_{L}(y, x) A_{L}^{\prime}\left(y^{\prime}, x\right),
$$

where the explicit expressions of the functions $A_{L}^{\left({ }^{\prime}\right)}$ are given by Eqs. (3.16a)- (3.17b) of Ref. [21]. The radial integration on $x$ is done and the functions $I^{(L)}$ are introduced,

$$
I^{(L)}\left(y, y^{\prime}\right)=\int_{0}^{1} x^{2} d x A_{L}(y, x) A_{L}^{\prime}\left(y^{\prime}, x\right) .
$$

The following expressions for the $s$ - and $p$-wave contributions to the EOS may be finally written:

$$
\begin{aligned}
& \frac{\Delta E_{\mathrm{sym}(l=1)}^{(2)}}{A}=-\frac{18 m k_{F}^{4}}{4 \pi^{4} \hbar^{2}} \sum_{S T}(2 T+1)(2 S+1) \delta_{S T 1} \iint d y d y^{\prime} \frac{y^{2} y^{\prime 2}}{y^{\prime 2}-y^{2}}\left[I^{(0)}\left(y, y^{\prime}\right)+2 I^{(2)}\left(y, y^{\prime}\right)\right]\left[v_{S, T, 1}\left(k_{F} y, k_{F} y^{\prime}\right)\right]^{2}, \\
& \frac{\Delta E_{\mathrm{sym}(l=0)}^{(2)}}{A}=-\frac{18 m k_{F}^{4}}{4 \pi^{4} \hbar^{2}} \sum_{S T} \delta_{S T 0} \iint d y d y^{\prime} \frac{y^{2} y^{\prime 2}}{y^{\prime 2}-y^{2}} I^{(0)}\left(y, y^{\prime}\right)\left[v_{S, T, 0}\left(k_{F} y, k_{F} y^{\prime}\right)\right]^{2} .
\end{aligned}
$$


The expressions of the EOS's may be then obtained analytically. The two terms that should be summed up are

$$
\begin{aligned}
\frac{\Delta E_{\mathrm{sym}(l=0)}^{(2)}}{A}= & -\frac{m k_{F}^{4}}{110880 \hbar^{2} \pi^{4}}\left\{\left[-6534+1188 \ln [2]+3564 \lambda-19602 \lambda^{3}-5940 \lambda^{5}+\left(1782-20790 \lambda^{4}\right) \ln \left[\frac{\lambda-1}{\lambda+1}\right]\right.\right. \\
& \left.+\left(24948 \lambda^{5}-5940 \lambda^{7}\right) \ln \left[\frac{\lambda^{2}-1}{\lambda^{2}}\right]\right] \widetilde{T}_{03}^{2}+\left[-14696+2112 \ln [2]+5280 \lambda-2860 \lambda^{3}-48840 \lambda^{5}\right. \\
& \left.-18480 \lambda^{7}+\left(2640-55440 \lambda^{6}\right) \ln \left[\frac{\lambda-1}{\lambda+1}\right]+\left(71280 \lambda^{7}-18480 \lambda^{9}\right) \ln \left[\frac{\lambda^{2}-1}{\lambda^{2}}\right]\right] k_{F}^{2} \widetilde{T}_{03} \widetilde{T}_{1} \\
& +\left[-9886+1128 \ln [2]+2520 \lambda+147 \lambda^{3}-3654 \lambda^{5}-35280 \lambda^{7}-15120 \lambda^{9}+\left(1260-41580 \lambda^{8}\right) \ln \left[\frac{\lambda-1}{\lambda+1}\right]\right. \\
& \left.\left.+\left(55440 \lambda^{9}-15120 \lambda^{11}\right) \ln \left[\frac{\lambda^{2}-1}{\lambda^{2}}\right]\right] k_{F}^{4} \widetilde{T}_{1}^{2}\right\}
\end{aligned}
$$

and

$$
\begin{aligned}
\frac{\Delta E_{\mathrm{sym}(l=1)}^{(2)}=}{A} & -\frac{m k_{F}^{8}}{73920 \hbar^{2} \pi^{4}}\left\{\left[-1033+156 \ln [2]+420 \lambda+140 \lambda^{3}-840 \lambda^{5}-5880 \lambda^{7}\right.\right. \\
& \left.\left.-2520 \lambda^{9}-\left(-210+6930 \lambda^{8}\right) \ln \left[\frac{\lambda-1}{\lambda+1}\right]+\left(9240 \lambda^{9}-2520 \lambda^{11}\right) \ln \left[\frac{\lambda^{2}-1}{\lambda^{2}}\right]\right] \widetilde{T}_{2}^{2}\right\},
\end{aligned}
$$

where we have introduced the combinations of parameters

$$
\begin{aligned}
\widetilde{T}_{03}^{2} & =\left[t_{0}\left(1-x_{0}\right)+\frac{1}{6} t_{3}\left(1-x_{3}\right) \rho^{\alpha}\right]^{2}+\left[t_{0}\left(1+x_{0}\right)+\frac{1}{6} t_{3}\left(1+x_{3}\right) \rho^{\alpha}\right]^{2}, \\
\widetilde{T}_{1}^{2} & =\frac{1}{4} t_{1}^{2}\left[\left(1-x_{1}\right)^{2}+\left(1+x_{1}\right)^{2}\right]=\frac{1}{2} t_{1}^{2}\left(1+x_{1}^{2}\right), \\
\widetilde{T}_{03} \widetilde{T}_{1} & =\frac{t_{1}}{2}\left[\left[t_{0}\left(1-x_{0}\right)+\frac{1}{6} t_{3}\left(1-x_{3}\right) \rho^{\alpha}\right]\left(1-x_{1}\right)+\left[t_{0}\left(1+x_{0}\right)+\frac{1}{6} t_{3}\left(1+x_{3}\right) \rho^{\alpha}\right]\left(1+x_{1}\right)\right], \\
\widetilde{T}_{2}^{2} & =\left[t_{2}^{2}\left(1-x_{2}\right)^{2}+9 t_{2}^{2}\left(1+x_{2}\right)^{2}\right] / 9=\frac{2}{9} t_{2}^{2}\left(5+8 x_{2}+5 x_{2}^{2}\right) .
\end{aligned}
$$

The asymptotic behavior can be written as a polynomial form in $\lambda$. One has to sum up the two terms

$$
\begin{aligned}
\frac{\Delta E_{\text {sym }(l=0), \text { asympt. }}^{(2)}=}{A} & -\frac{9 m k_{F}^{4}}{2 \hbar^{2} \pi^{4}}\left[\frac{k_{F}^{4} \widetilde{T}_{1}^{2}}{360} \lambda^{5}+\left(\frac{k_{F}^{2} \widetilde{T}_{03} \widetilde{T}_{1}}{108}+\frac{k_{F}^{4} \widetilde{T}_{1}^{2}}{240}\right) \lambda^{3}+\left(\frac{\widetilde{T}_{03}^{2}}{72}+\frac{k_{F}^{2} \widetilde{T}_{03} \widetilde{T}_{1}}{60}+\frac{k_{F}^{4} \widetilde{T}_{1}^{2}}{140}\right) \lambda\right. \\
& +\frac{44 k_{F}^{2} \widetilde{T}_{03} \widetilde{T}_{1}(-167+24 \ln [2])+k_{F}^{4} \widetilde{T}_{1}^{2}(-4943+564 \ln [2])+297 \widetilde{T}_{03}^{2}(-11+2 \ln [2])}{249480} \\
& \left.-\left(\frac{\widetilde{T}_{03}^{2}}{240}+\frac{k_{F}^{2} \widetilde{T}_{03} \widetilde{T}_{1}}{140}+\frac{k_{F}^{4} \widetilde{T}_{1}^{2}}{270}\right) / \lambda+O\left(\lambda^{-2}\right)\right]
\end{aligned}
$$

and

$$
\frac{\Delta E_{\text {sym }(l=1) \text {,asympt. }}^{(2)}}{A}=-\frac{9 m k_{F}^{8}}{2 \hbar^{2} \pi^{4}}\left[\frac{1}{720} \lambda^{3}+\frac{1}{560} \lambda+\left(\frac{-1033+156 \ln [2]}{332640}\right)-\left(\frac{1}{1080}\right) / \lambda+O\left(\lambda^{-2}\right)\right] \widetilde{T}_{2}^{2} .
$$

\section{Neutron matter}

Note that the triple integral is the same for neutron and symmetric matter. The factors are not the same (see the $n n$ contribution in the Appendix), leading to a different combination of the Skyrme parameters, and $k_{F} \rightarrow k_{F_{N}}$. Here we report the final result. One has to sum up the two terms

$$
\begin{aligned}
\frac{\Delta E_{\text {neutr }(l=0)}^{(2)}=}{A} & -\frac{m k_{F_{N}}^{4}}{166320 \hbar^{2} \pi^{4}}\left\{\left[-6534+1188 \ln [2]+3564 \lambda-19602 \lambda^{3}-5940 \lambda^{5}+\left(1782-20790 \lambda^{4}\right) \ln \left[\frac{\lambda-1}{\lambda+1}\right]\right.\right. \\
& \left.+\left(24948 \lambda^{5}-5940 \lambda^{7}\right) \ln \left[\frac{\lambda^{2}-1}{\lambda^{2}}\right]\right] T_{03}^{2}+\left[-14696+2112 \ln [2]+5280 \lambda-2860 \lambda^{3}-48840 \lambda^{5}\right.
\end{aligned}
$$




$$
\begin{aligned}
& \left.-18480 \lambda^{7}+\left(2640-55440 \lambda^{6}\right) \ln \left[\frac{\lambda-1}{\lambda+1}\right]+\left(71280 \lambda^{7}-18480 \lambda^{9}\right) \ln \left[\frac{\lambda^{2}-1}{\lambda^{2}}\right]\right] k_{F_{N}}^{2} T_{03} T_{1} \\
& +\left[-9886+1128 \ln [2]+2520 \lambda+147 \lambda^{3}-3654 \lambda^{5}-35280 \lambda^{7}-15120 \lambda^{9}+\left(1260-41580 \lambda^{8}\right) \ln \left[\frac{\lambda-1}{\lambda+1}\right]\right. \\
& \left.\left.+\left(55440 \lambda^{9}-15120 \lambda^{11}\right) \ln \left[\frac{\lambda^{2}-1}{\lambda^{2}}\right]\right] k_{F_{N}^{4}}^{4} T_{1}^{2}\right\}
\end{aligned}
$$

and

$$
\begin{aligned}
\frac{\Delta E_{\text {neutr }(l=1)}^{(2)}=}{A} & -\frac{m k_{F_{N}}^{8}}{110880 \hbar^{2} \pi^{4}}\left\{\left[-1033+156 \ln [2]+420 \lambda+140 \lambda^{3}-840 \lambda^{5}-5880 \lambda^{7}-2520 \lambda^{9}\right.\right. \\
& \left.\left.-\left(-210+6930 \lambda^{8}\right) \ln \left[\frac{\lambda-1}{\lambda+1}\right]+\left(9240 \lambda^{9}-2520 \lambda^{11}\right) \ln \left[\frac{\lambda^{2}-1}{\lambda^{2}}\right]\right] T_{2}^{2}\right\},
\end{aligned}
$$

where now the combinations of parameters are defined as

$$
\begin{aligned}
T_{03} & =t_{0}\left(1-x_{0}\right)+\frac{1}{6} t_{3}\left(1-x_{3}\right) \rho^{\alpha}, \\
T_{1} & =\frac{1}{2} t_{1}\left(1-x_{1}\right), \\
T_{2} & =t_{2}\left(1+x_{2}\right) .
\end{aligned}
$$

The asymptotic behavior is written as the sum of the two terms

$$
\begin{aligned}
\frac{\Delta E_{\text {neutr }(l=0) \text { asympt. }}^{(2)}=}{A} & -\frac{3 m k_{F_{N}}^{4}}{\hbar^{2} \pi^{4}}\left[\frac{k_{F_{N}}^{4} T_{1}^{2}}{360} \lambda^{5}+\left(\frac{k_{F_{N}}^{2} T_{03} T_{1}}{108}+\frac{k_{F_{N}}^{4} T_{1}^{2}}{240}\right) \lambda^{3}+\left(\frac{T_{03}^{2}}{72}+\frac{k_{F_{N}}^{2} T_{03} T_{1}}{60}+\frac{k_{F_{N}}^{4} T_{1}^{2}}{140}\right) \lambda\right. \\
& +\frac{44 k_{F_{N}}^{2} T_{03} T_{1}(-167+24 \ln [2])+k_{F_{N}}^{4} T_{1}^{2}(-4943+564 \ln [2])+297 T_{03}^{2}(-11+2 \ln [2])}{249480} \\
& \left.-\left(\frac{T_{03}^{2}}{240}+\frac{k_{F_{N}}^{2} T_{03} T_{1}}{140}+\frac{k_{F_{N}}^{4} T_{1}^{2}}{270}\right) / \lambda+O\left(\lambda^{-2}\right)\right]
\end{aligned}
$$

and

$$
\frac{\Delta E_{\text {neutr }(l=1) \text {,asympt. }}^{(2)}}{A}=-\frac{3 m k_{F_{N}}^{8}}{\hbar^{2} \pi^{4}}\left[\frac{1}{720} \lambda^{3}+\frac{1}{560} \lambda+\left(\frac{-1033+156 \ln [2]}{332640}\right)-\left(\frac{1}{1080}\right) / \lambda+O\left(\lambda^{-2}\right)\right] T_{2}^{2} .
$$

One may note that in both EOS's (symmetric and neutron matter) the divergence is linear in $\lambda$ if only the $t_{0}-t_{3}$ part of the interaction is taken, and goes like $\lambda^{5}$ if the other terms of the interaction are also included, as was already indicated in Refs. [8,9]. The strongest divergence is provided by the $t_{1}$ term.

\section{B. Numerical results and fits of parameters for symmetric, asymmetric, and pure neutron matter}

We solve the second-order integrals for symmetric, neutron, and asymmetric matter in the illustrative case $\delta=0.5$, where $\delta$ is the asymmetry parameter $\delta=\left(\rho_{n}-\rho_{p}\right) /\left(\rho_{n}+\rho_{p}\right)$ and $\rho_{n}$ and $\rho_{p}$ are the neutron and proton densities, respectively. Several types of integrals are solved numerically (according to the specific second-order contribution). The factors for which such integrals are multiplied are shown in the Appendix for all terms.

The adjustment of the parameters is done in all cases on the benchmark SLy5-mean-field EOS [22]; we use nine points, seven of them located up to $0.16 \mathrm{fm}^{-3}$ and two of them located at higher densities, between 0.16 and $0.3 \mathrm{fm}^{-3}$.
The $\chi^{2}$ values are calculated as $\chi^{2}=1 /(N-1) \sum_{i}\left(E_{i}-\right.$ $\left.E_{i, \text { ref }}\right)^{2} / \Delta E_{i}^{2}$, where $N$ is the number of points on which the

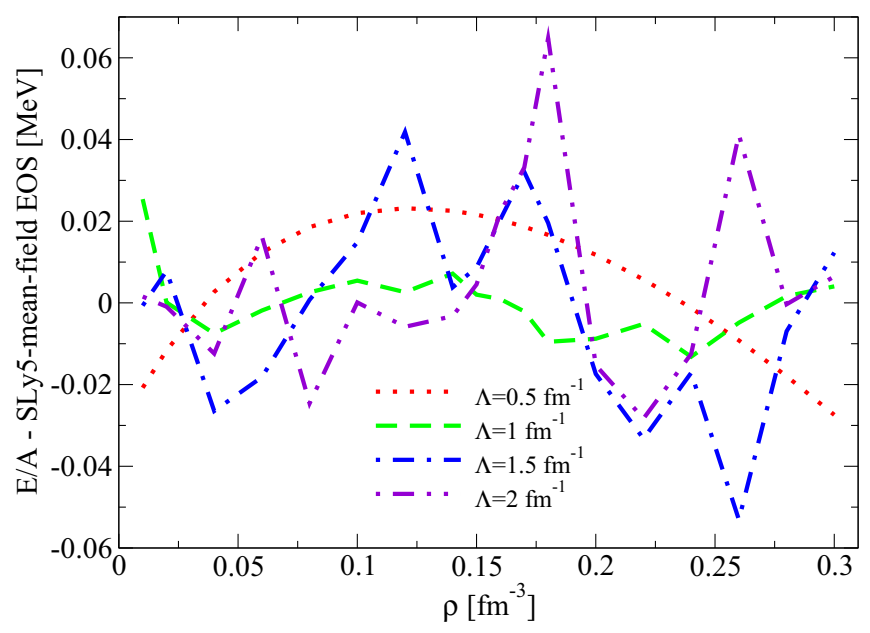

FIG. 8. Difference between the refitted EOS and the SLy5-meanfield EOS for neutron matter. 


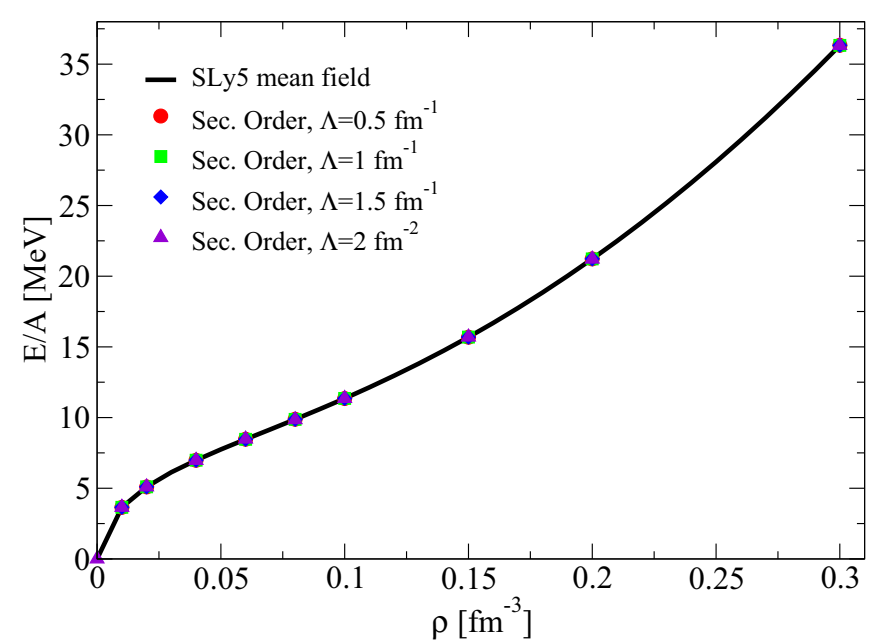

FIG. 9. Refitted second-order EOS for neutron matter compared with the SLy5-mean-field EOS.

adjustment is done (the sum runs over this number), $E_{i \text {, ref }}$ is the benchmark value corresponding to the point $i$, and $\Delta E_{i}$ are all chosen equal to $1 \%$ of the reference value. This means that, if the $\chi^{2}$ is less than 1 , the average discrepancy between the adjusted curve and the benchmark EOS is less than $1 \%$.

\section{Symmetric matter and incompressibility modulus}

We plot in the upper panel of Fig. 1 the EOS of symmetric matter calculated up to second order for several cutoff values $\Lambda$. These curves are compared to the benchmark EOS. All the second-order curves are obtained by using the same parameters of SLy5. In the lower panel, we plot only the second-order correction. The ultraviolet divergence is very visible, especially at densities larger than the saturation density. Starting from some values of the cutoff between 1.5

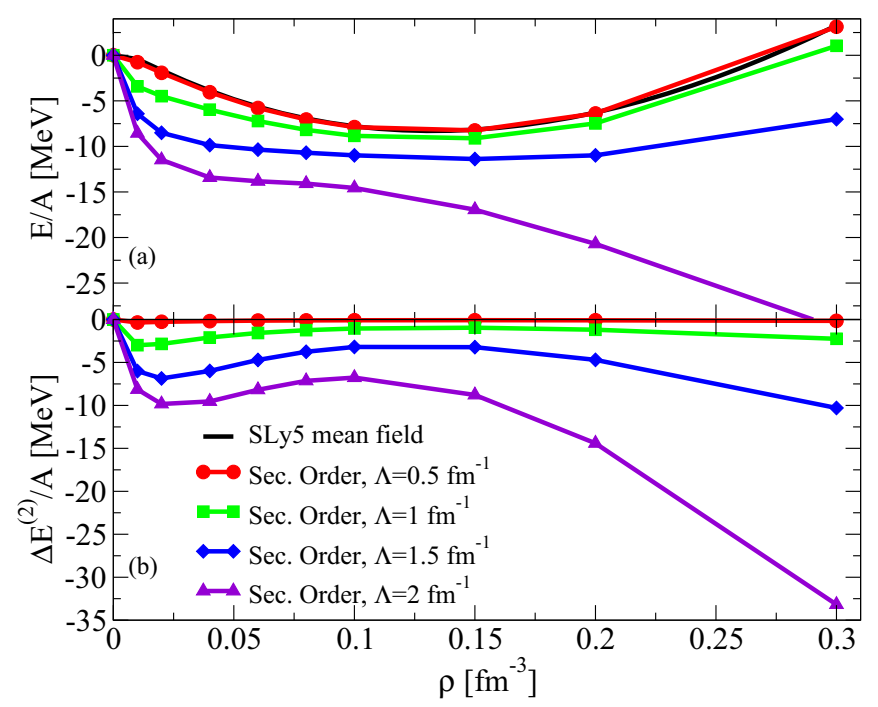

FIG. 10. (a) Second-order EOS of asymmetric matter $(\delta=0.5)$ for several values of the cutoff, calculated with the SLy5 parameters, and SLy5-mean-field EOS; (b) second-order correction.

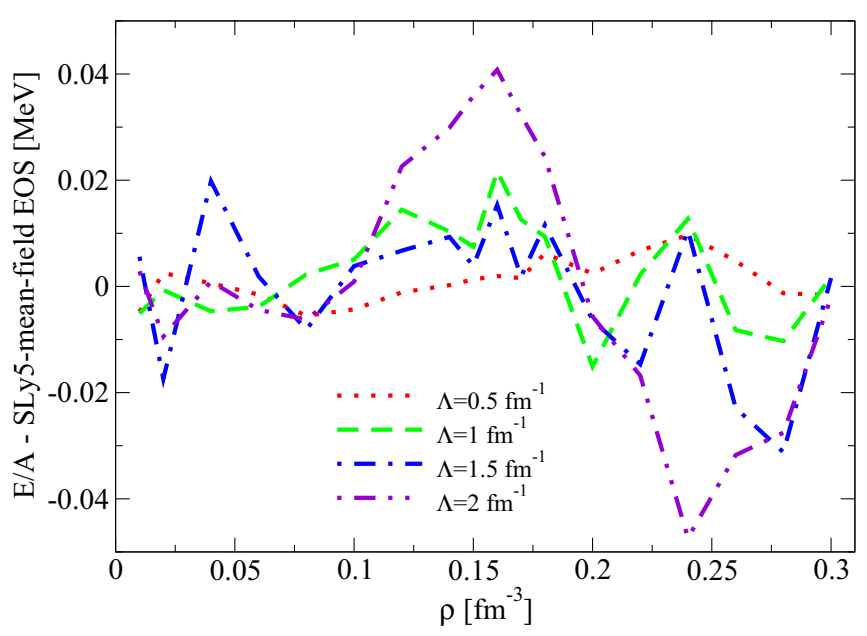

FIG. 11. Difference between the refitted EOS and the SLy5mean-field EOS for asymmetric matter $(\delta=0.5)$.

and $2 \mathrm{fm}^{-1}$, one observes that the EOS decreases (instead of increasing) at large densities.

The pressure $P$ and the incompressibility modulus $K$ may be computed from the EOS as first and second derivatives, respectively, that is,

$$
P(\rho, \Lambda)=\rho^{2} \frac{d}{d \rho} \frac{E}{A}(\rho, \Lambda)
$$

and

$$
K(\rho, \Lambda)=9 \rho^{2} \frac{d^{2}}{d \rho^{2}} \frac{E}{A}(\rho, \Lambda) .
$$

The second-order pressure (upper panel) and incompressibility modulus (lower panel) calculated with the parameters of the interaction SLy5 are displayed in Fig. 2 and compared with the corresponding mean-field curves.

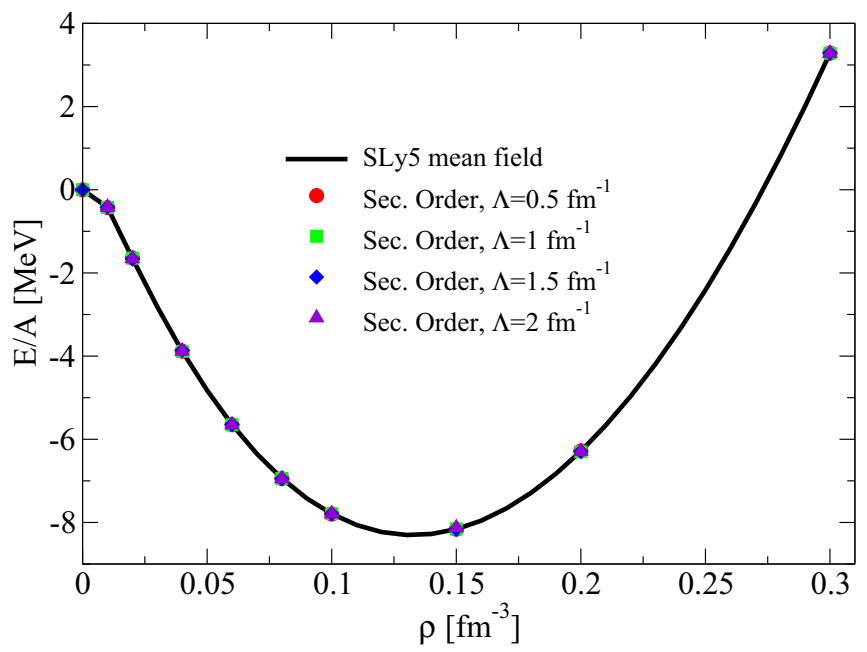

FIG. 12. Refitted second-order EOS for asymmetric matter $(\delta=$ 0.5) compared with the SLy5-mean-field EOS. 
TABLE III. Parameter sets obtained with the fit of the second-order EOS of asymmetric matter $(\delta=0.5)$ matter for different values of the cutoff $\Lambda$ compared with the original set SLy5. The $\chi^{2}$ values are shown in the last column.

\begin{tabular}{|c|c|c|c|c|c|c|c|c|c|c|}
\hline $\begin{array}{l}\text { SLy5 } \\
\Lambda\left(\mathrm{fm}^{-1}\right)\end{array}$ & -2484.88 & 483.13 & -549.40 & 13763.0 & 0.778 & -0.328 & -1.0 & 1.267 & 0.16667 & \\
\hline 0.5 & -1639.753 & 584.294 & -732.339 & 19750.210 & 0.5989 & -0.339 & -0.886 & 3.328 & 0.284 & $4.76 \times 10^{-3}$ \\
\hline 1.0 & -1252.011 & 558.930 & -744.859 & 10167.053 & 0.5887 & -0.4652 & -1.018 & 2.853 & 0.2272 & $1.19 \times 10^{-2}$ \\
\hline
\end{tabular}

The adjustment of the nine Skyrme parameters is then performed and Figs. 3 and 4 show the curves obtained with the refitted parameters. Figure 3 presents the difference between the refitted second-order curve and the SLy5-mean-field curve, whereas the absolute values are displayed in Fig. 4. The saturation density is, for all values of the cutoff, the same as the benchmark one; that is, $0.16 \mathrm{fm}^{-3}$. The refitted parameters are listed in Table I together with the $\chi^{2}$ values. The quality of the fit looks remarkably good as indicated by the $\chi^{2}$ values which are, in all cases, not larger than $\sim 3 \times 10^{-2}$.

The pressure and incompressibility modulus are then computed at second order, this time with the new values for the parameters. Figure 5 displays the difference with respect to the mean-field curves for the pressure (a) and the incompressibility modulus (b). Figure 6 shows the absolute curves compared with the mean-field ones. We observe that the maximum deviation of the incompressibility modulus from the SLy5-mean-field value at the saturation density $(\sim 230 \mathrm{MeV})$ is only $\sim 5 \mathrm{MeV}$. This deviation is indicated by a black arrow in the lower panel of Fig. 5. From the upper panel one can note

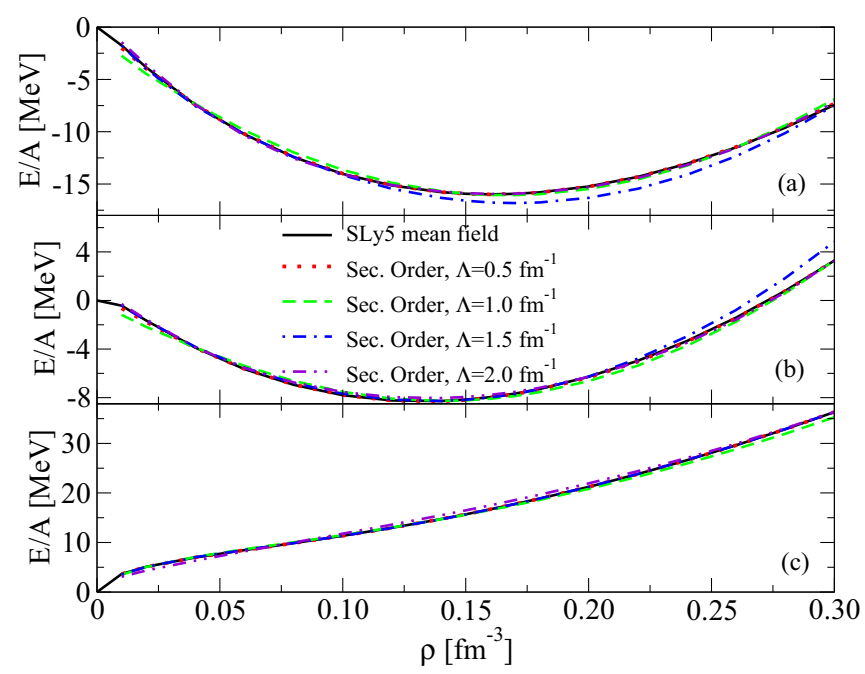

FIG. 13. Second-order EOS's obtained with the simultaneous fit of symmetric matter (a), asymmetric matter with $\delta=0.5$ (b), and neutron matter (c) compared with the SLy5-mean-field corresponding curves. that the pressure is non strictly equal to zero at the saturation density. The deviation in the derivative is, however, very small and accounts for very small variations in the saturation density for the different fits.

\section{Neutron matter}

We show in Fig. 7 the second-order EOS for pure neutron matter (a) and the second-order correction (b). In Table II we list the values of the refitted parameters. Figures 8 and 9 show the refitted results (differences with respect to the mean-field curve and absolute values, respectively). The fit is also this time extremely good, and the $\chi^{2}$ values are of the order of $10^{-2}$.

\section{Asymmetric matter in the case of $\delta=0.5$}

For asymmetric matter we take the illustrative case corresponding to $\delta=0.5$. Figure 10 shows the second-order EOS (a) and the second-order correction (b). Figures 11 and 12 present the refitted results shown again as differences with respect to the benchmark EOS (Fig. 11) and as absolute values (Fig. 12). Table III contains the refitted parameters and the $\chi^{2}$ values, which range from $10^{-3}$ to $10^{-1}$, according to the value

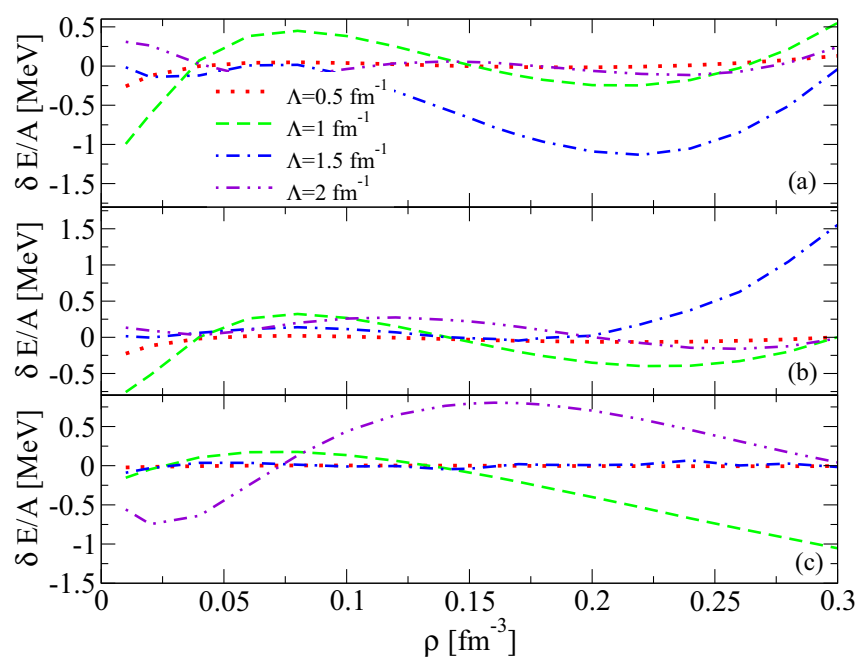

FIG. 14. Difference between the global refitted EOS's and the SLy5-mean-field EOS's for symmetric (a), $\delta=0.5$ (b), and neutron (c) matter. 
TABLE IV. Parameter sets obtained with the global fit of the second-order EOS including symmetric, $\delta=0.5$ and neutron matter for different values of the cutoff $\Lambda$ compared with the original set SLy5. The $\chi^{2}$ values are shown in the last column.

\begin{tabular}{|c|c|c|c|c|c|c|c|c|c|c|}
\hline $\begin{array}{l}\text { SLy5 } \\
\Lambda\left(\mathrm{fm}^{-1}\right)\end{array}$ & -2484.88 & 483.13 & -549.40 & 13736.0 & 0.778 & -0.328 & -1.0 & 1.267 & 0.16667 & \\
\hline 0.5 & -2245.402 & 493.322 & -1832.783 & 11961.86 & 0.7462 & -0.3936 & -0.9684 & 1.309 & 0.1832 & 0.25 \\
\hline 1.0 & -1239.909 & 674.272 & -387.948 & 4687.107 & 0.3649 & -0.5993 & -1.1349 & 3.4299 & 0.5558 & 3.96 \\
\hline
\end{tabular}

of the cutoff. The $\chi^{2}$ values are still lower than 1 . We can conclude that the quality of the fit is always extremely good in the three cases of symmetric, neutron, and asymmetric matter.

\section{Simultaneous fit of symmetric, neutron, and asymmetric matter in the case $\delta=0.5$}

We have then adjusted simultaneously the second-order EOS's of symmetric, asymmetric $(\delta=0.5)$, and neutron matter, as was done in Ref. [9]. The obtained curves are shown in Figs. 13 and 14 in absolute values and as differences with respect to the benchmark EOS's, respectively. The values of the adjusted parameters are reported in Table IV with the associated $\chi^{2}$ values. We observe that now the deviations from the benchmark EOS's are larger than in the previous single fits providing however acceptable EOS's in all cases. The average discrepancy is less than $2 \%$ for cutoff values of 0.5 and $1 \mathrm{fm}^{-1}$ ( $\chi^{2}$ values equal to 0.25 and 3.96 , respectively), and is $\sim 3.5 \%$ for cutoff values of 1.5 and $2 \mathrm{fm}^{-1}$ ( $\chi^{2}$ values equal to 13.9 and 10.7, respectively). The corresponding pressure and incompressibility modulus are shown in absolute values and as differences with respect to the mean-field curves in Figs. 15 and 16, respectively. The incompressibility modulus at the saturation point has a maximum deviation from the mean-field

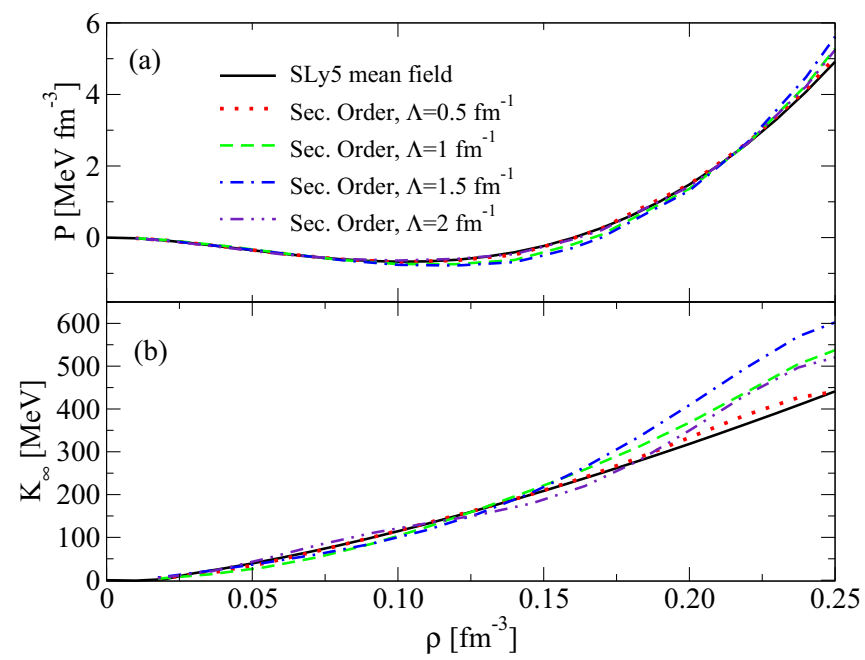

FIG. 15. Pressure (a) and incompressibility modulus (b) computed with the parameters of the simultaneous fit and compared with the mean-field curves. value of $\sim 25 \mathrm{MeV}$, as indicated by the black arrows in the lower panel of Fig. 16. Also in this case the pressure is not strictly equal to zero at saturation. The deviation is now larger than in the previous case (larger deviations in the saturation density for the different fits).

\section{DIMENSIONAL REGULARIZATION}

In the present section, we report the revised results, with respect to Ref. [10], concerning the application of the dimensional regularization to the second-order integrals and the extraction of the corresponding finite contributions in the EOS's of symmetric, neutron, and asymmetric matter, for the case $\delta=0.5$. This regularization technique, that was originally introduced for the electroweak theory [23-25], is based on the solution of the divergent integrals with the use a continuous parameter $d$ which replaces their integer dimension. After the evaluation of the integral, the parameter $d$ returns to the integer value.

In Ref. [10], the first analyzed case was the simple model $t_{0}-t_{3}$ applied to symmetric matter (the case analogous to that of Ref. [8]). This was correctly treated and the corresponding results are reported in Secs. II A and III A and up to Fig. 2 of Ref. [10].

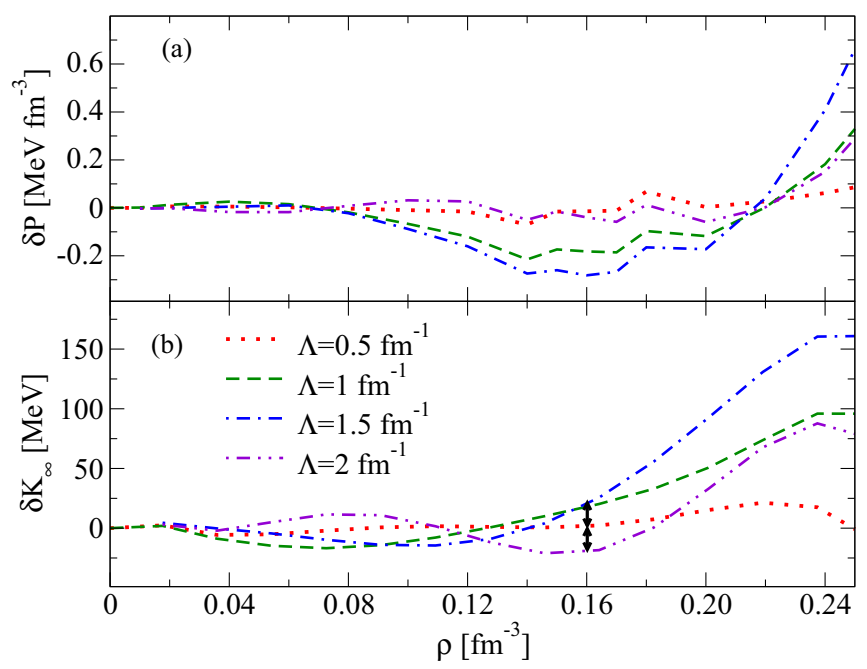

FIG. 16. Differences with respect to the mean-field curves of the pressure (a) and incompressibility modulus (b), computed with the parameters of the simultaneous fit. 


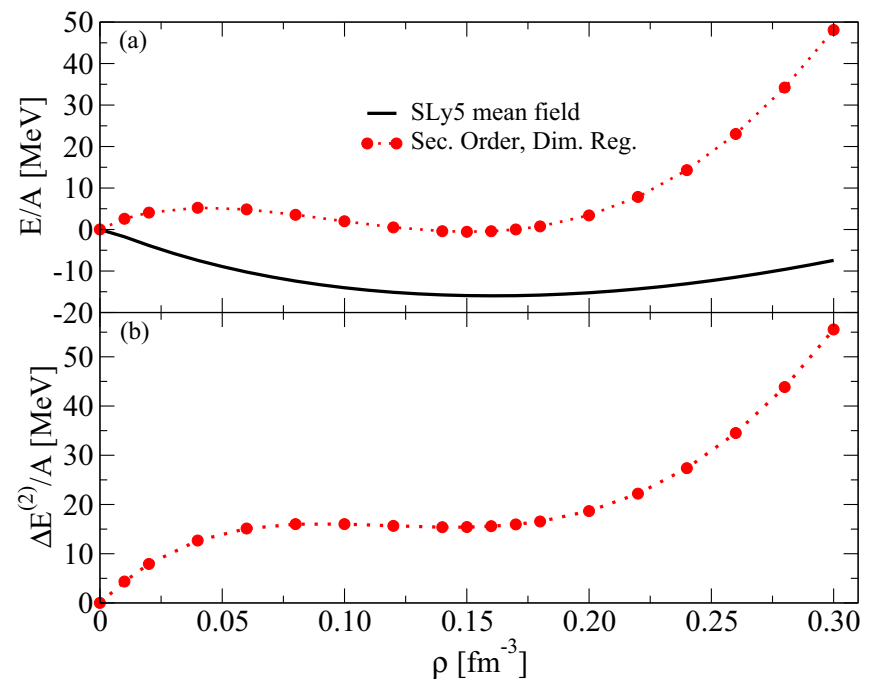

FIG. 17. (a) Dimensional-regularized second-order EOS for symmetric matter compared with the corresponding SLy5-mean-field EOS. (b) Second-order correction.

For the other cases (Skyrme interaction containing all terms except the tensor and the spin-orbit parts, and the treatment of also asymmetric and neutron matter) the same corrections done in the previous section have to be performed in the evaluation of the second-order contribution. We provide in what follows the analytical expressions of the second-order contributions for symmetric and neutron matter. We analyze the results for symmetric, neutron, and asymmetric matter in the case $\delta=$ 0.5 . The case of asymmetric matter is not derived analytically, but the finite part of this EOS is extracted from the numerical Monte Carlo calculation.

Also in the present section we employ the approximation $m^{*}=m$ and we neglect the rearrangement terms at second order. These approximations will be abandoned in Sec. IV.

\section{A. Symmetric matter}

Starting from the Skyrme interaction of Eq. (1), the dimensional-regularized second-order result for symmetric matter is written as

$$
\begin{aligned}
\frac{E_{\mathrm{sym}}^{(2) F}}{A}= & \frac{m k_{F}^{4}}{\hbar^{2} \pi^{4}}\left(\frac{3}{560}(11-2 \ln [2]) \widetilde{T}_{03}^{2}\right. \\
& +\frac{1}{1260}(167-24 \ln [2]) k_{F}^{2} \widetilde{T}_{03} \widetilde{T}_{1}
\end{aligned}
$$

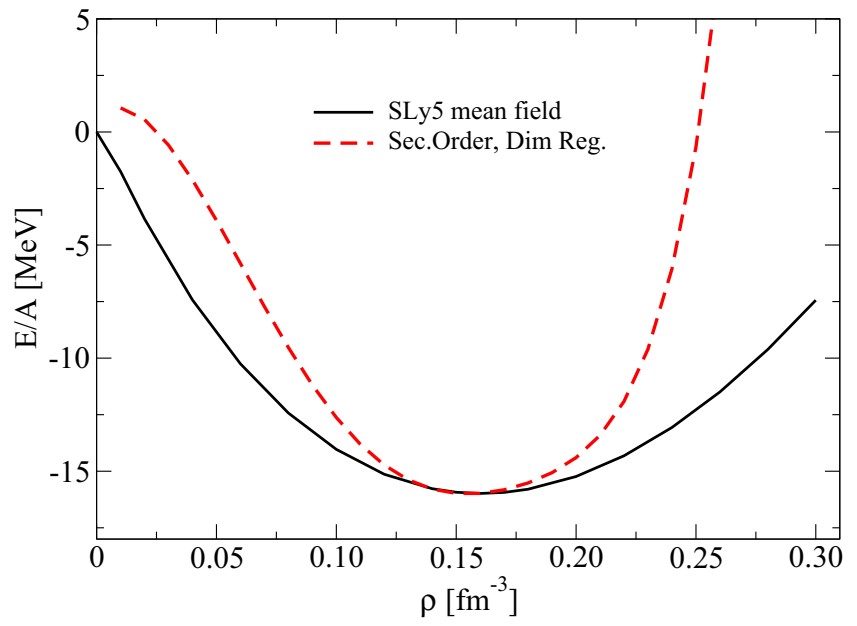

FIG. 18. Refitted dimensional-regularized second-order EOS for symmetric matter compared with the corresponding SLy5-mean-field EOS.

$$
\begin{aligned}
& +\frac{1}{55440}(4943-564 \ln [2]) k_{F}^{4} \widetilde{T}_{1}^{2} \\
& \left.+\frac{1}{73920}(1033-156 \ln [2]) k_{F}^{4} \widetilde{T}_{2}^{2}\right),
\end{aligned}
$$

where $F$ stands for finite part. The second-order EOS compared with the SLy5-mean-field EOS is displayed in Fig. 17. The refitted curve is plotted in Fig. 18 and the corresponding parameters are written in Table $\mathrm{V}(\delta=0)$.

We could correctly reproduce the saturation region only by restricting the fit of the parameters in a narrow region of densities close to the saturation point. The EOS is, however, poorly described in the other density regions, as can be seen in Fig. 18; this implies that the incompressibility modulus is not well reproduced $(368 \mathrm{MeV})$. An improvement is however seen with respect to the adjustment presented in Ref. [13], where the equilibrium point was completely missed. This improvement is due to the density-dependent term which allows us at least to shift the equilibrium point to its correct value. We do not report for this case the $\chi^{2}$ value because the reference points for the fit are taken only close to the saturation point and such $\chi^{2}$ value would thus not be comparable with those obtained from

\begin{tabular}{|c|c|c|c|c|c|c|c|c|c|}
\hline $\begin{array}{l}\text { SLy5 } \\
\delta\end{array}$ & -2484.88 & 483.13 & -549.40 & 13763.0 & 0.778 & -0.328 & -1.0 & 1.267 & 0.16667 \\
\hline 1 & -3746.7 & 264.38 & 1607.4 & -4537.9 & 0.8322 & -1.3524 & -1.1643 & -13.7421 & 2.0301 \\
\hline 0.5 & -920.60 & 544.55 & -783.28 & -879958 & 0.0289 & -0.2788 & -0.0681 & -10650 & 9.1666 \\
\hline
\end{tabular}
the previous fits where the reference points were distributed in the whole region of densities.

TABLE V. Parameter sets obtained with the fit of the dimensional-regularized second-order EOS compared with the original set SLy5. 


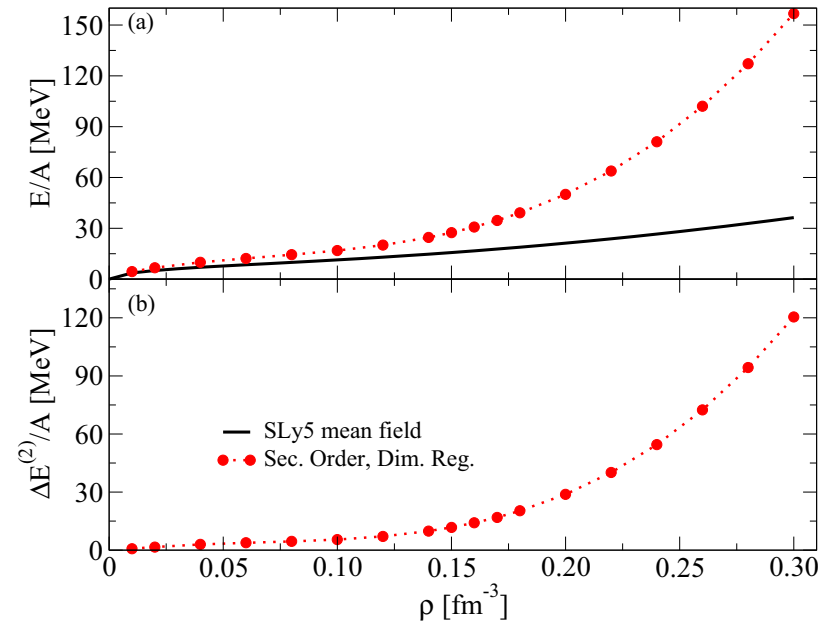

FIG. 19. (a) Dimensional regularized second-order EOS for neutron matter compared with the corresponding SLy5-mean-field EOS. (b) Second-order correction.

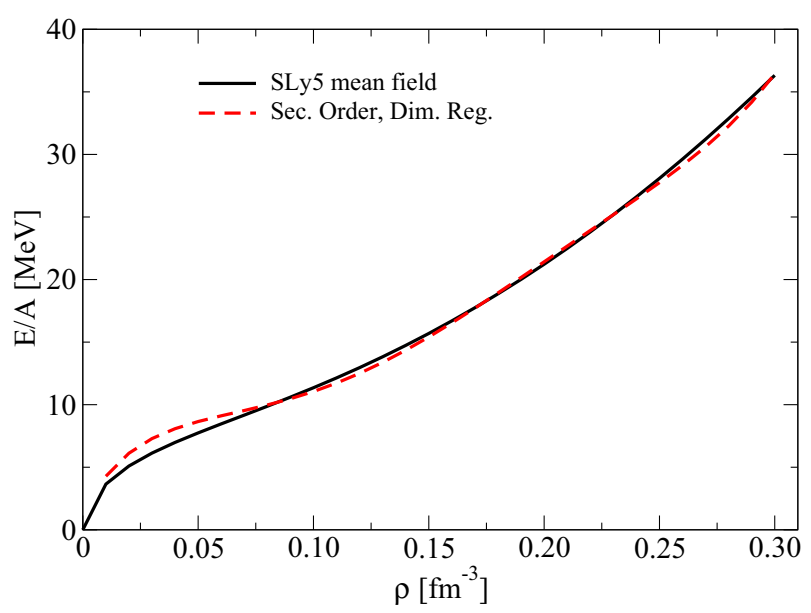

FIG. 20. Best fit for dimensional regularized second-order EOS of neutron matter compared with the SLy5-mean-field EOS.

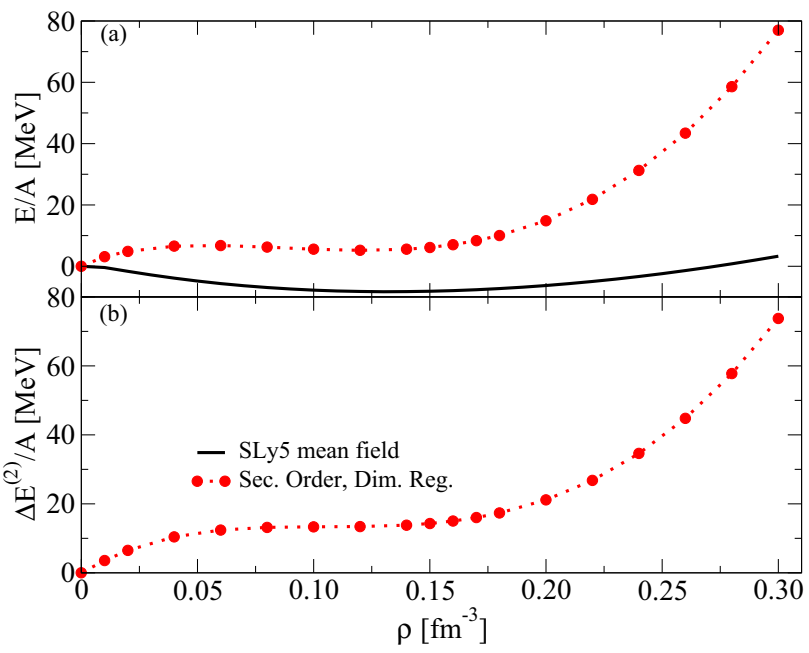

FIG. 21. (a) Dimensional-regularized second-order EOS for asymmetric matter in the case $\delta=0.5$ compared with the corresponding SLy5-mean-field EOS. (b) Second-order correction.

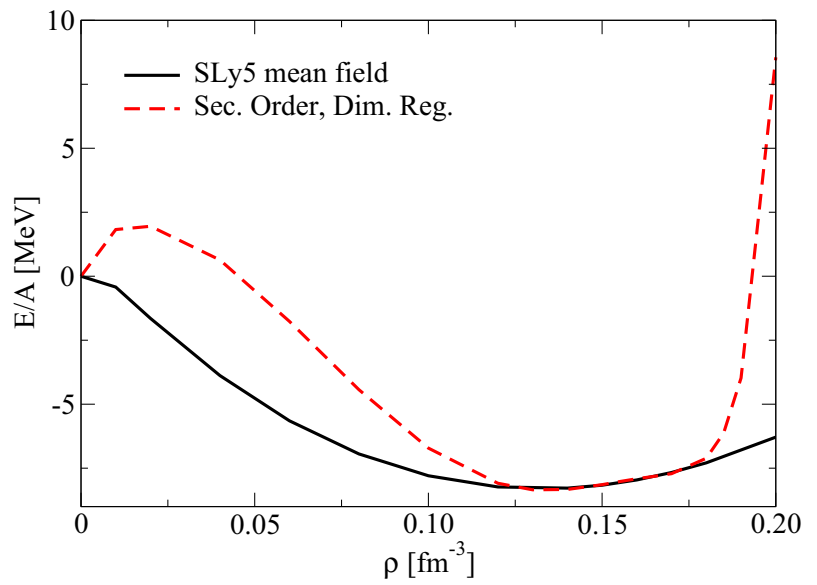

FIG. 22. Best fit for dimensional-regularized second-order EOS for asymmetric matter $(\delta=0.5)$ matter compared with the SLy5mean-field EOS.

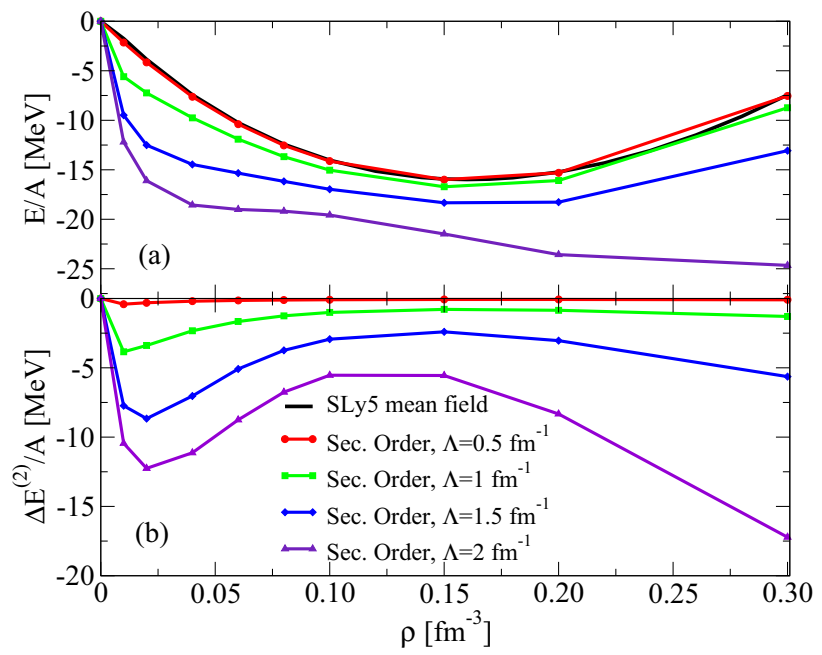

FIG. 23. Same as Fig. 1 but with an effective mass computed within the mean-field approximation.

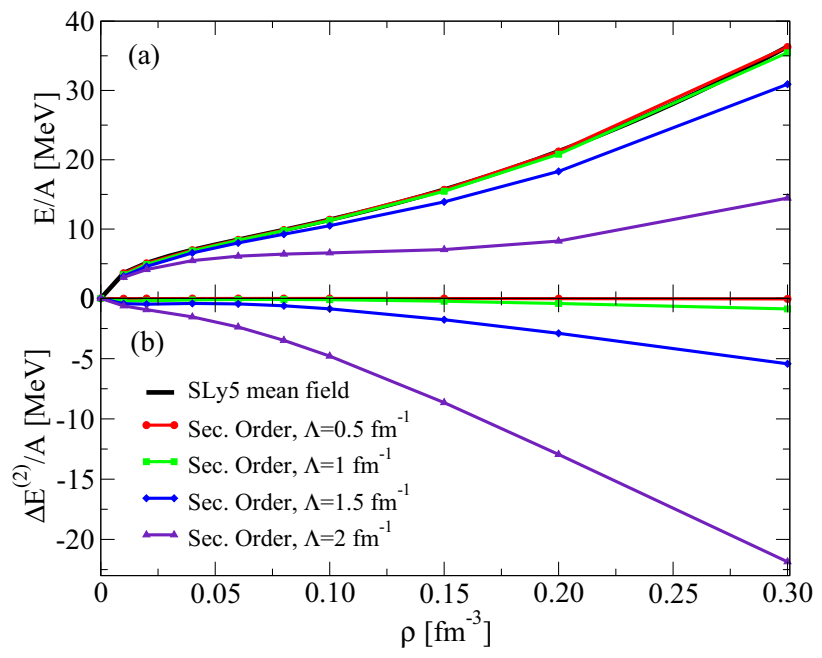

FIG. 24. Same as Fig. 7 but with an effective mass computed within the mean-field approximation. 
TABLE VI. Parameter sets obtained with the global fit of the second-order EOS including symmetric and neutron matter for different values of the cutoff $\Lambda$ compared with the original set SLy5. Here the mean-field effective mass is used in the second-order calculation. The $\chi^{2}$ values are shown in the last column.

\begin{tabular}{|c|c|c|c|c|c|c|c|c|c|c|}
\hline & $\begin{array}{c}t_{0} \\
\left(\mathrm{MeV} \mathrm{fm}^{3}\right)\end{array}$ & $\begin{array}{c}t_{1} \\
\left(\mathrm{MeV} \mathrm{fm}^{5}\right)\end{array}$ & $\begin{array}{c}t_{2} \\
\left(\mathrm{MeV} \mathrm{fm}^{5}\right)\end{array}$ & $\begin{array}{c}t_{3} \\
\left(\mathrm{MeV} \mathrm{fm}^{3+3 \alpha}\right)\end{array}$ & $x_{0}$ & $x_{1}$ & $x_{2}$ & $x_{3}$ & $\alpha$ & $\chi^{2}$ \\
\hline $\begin{array}{l}\text { SLy5 } \\
\Lambda\left(\mathrm{fm}^{-1}\right)\end{array}$ & -2484.88 & 483.13 & -549.40 & 13736.0 & 0.778 & -0.328 & -1.0 & 1.267 & 0.16667 & \\
\hline 0.5 & -2254.55 & 555.99 & -496.33 & 12099.62 & 0.7429 & -0.3723 & -0.9236 & 1.2982 & 0.1844 & 0.39 \\
\hline 1.0 & -1090.87 & 290.62 & -552.05 & 11613.08 & 0.2255 & -5.3624 & -0.8087 & 2.1436 & 0.6404 & 10 \\
\hline 1.5 & -433.47 & 203.22 & -280.96 & 6704.25 & 0.3570 & -5.4828 & -0.9308 & 2.7269 & 0.8262 & 1.9 \\
\hline
\end{tabular}

\section{B. Neutron matter}

The analytical expression for the second-order contribution is

$$
\begin{aligned}
\frac{E_{\text {neutr }}^{(2) F}}{A}= & \frac{m k_{N}^{4}}{\hbar^{2} \pi^{4}}\left(\frac{1}{280}(11-2 \ln [2]) T_{03}^{2}\right. \\
& +\frac{1}{1890}(167-24 \ln [2]) k_{N}^{2} T_{03} T_{1} \\
& +\frac{1}{83160}(4943-564 \ln [2]) k_{N}^{4} T_{1}^{2} \\
& \left.+\frac{1}{110880}(1033-156 \ln [2]) k_{N}^{4} T_{2}^{2}\right)
\end{aligned}
$$

The corresponding EOS is plotted in Fig. 19 and compared with the corresponding SLy5-mean-field EOS. Figure 20 shows the curve obtained with the adjusted parameters and Table $\mathrm{V}$ presents the associated parameters $(\delta=1)$. In this case, the nine reference points chosen for the fit are distributed in the whole region of densities. The $\chi^{2}$ value is $\sim 91$, indicating an average deviation from the reference points of about $9.5 \%$. The quality of the fit is lower than that obtained

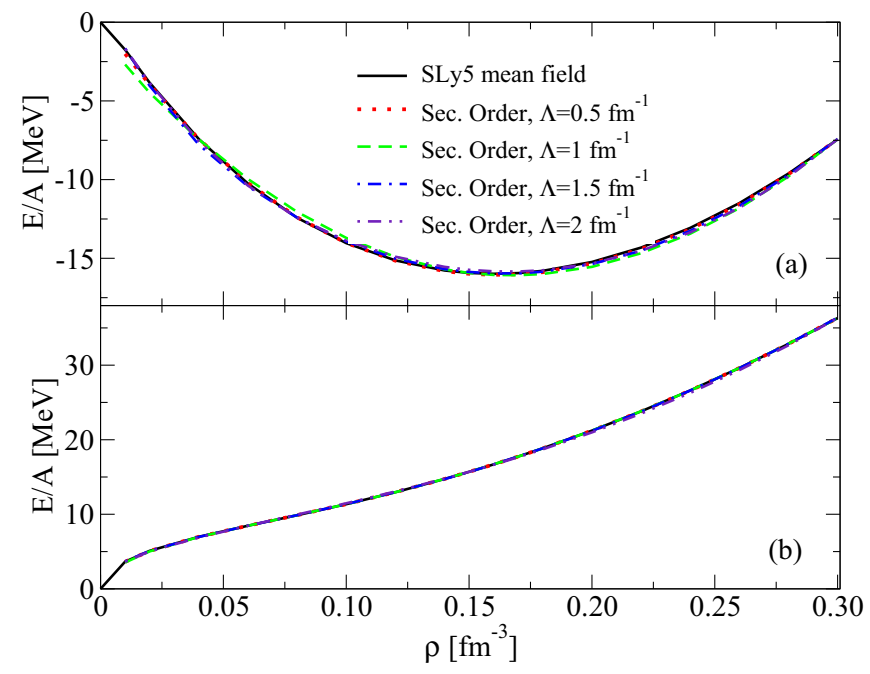

FIG. 25. Second-order EOS's for symmetric (a) and pure neutron matter (b) adjusted on the SLy5-mean-field EOS's, with an effective mass equal to the mean-field effective mass, for different values of the cutoff. for neutron matter in the case of the cutoff regularization, but is still reasonably good.

\section{Asymmetric matter}

The second-order EOS of asymmetric matter (for the case $\delta=0.5$ ) and the refitted curve are plotted respectively in Figs. 21 and 22. The parameters are listed in Table V. Also in this case, as for symmetric matter, the equilibrium region can be described only by taking a narrow region of densities around the minimum to perform the adjustment of the parameters. The EOS is clearly very poorly described in the other density regions.

We may conclude that, when dimensional regularization is used, the fit of the parameters has a global good quality only for the case of pure neutron matter. We will see, however, in the next section that results are considerably improved for symmetric matter when the rearrangement terms are taken into account.

\section{EFFECTIVE MASS AND REARRANGEMENT TERMS}

We worked so far by using two approximations: We have approximated the effective mass with the bare mass and we have neglected the second-order rearrangement terms generated by the density-dependent part of the interaction. In the present section, we include first an effective mass in the computation of the second-order EOS for the cases of symmetric and pure neutron matter. Then, we also include in these cases the corresponding rearrangement terms.

We use the mean-field approximation for the effective mass, where only the velocity-dependent terms contribute. For symmetric matter, one has

$$
\frac{m_{S}^{*}}{m}=\left(1+\frac{m}{8 \hbar^{2}} \rho \Theta_{S}\right)^{-1}
$$

whereas for neutron matter one has

$$
\frac{m_{N}^{*}}{m}=\left(1+\frac{m}{4 \hbar^{2}} \rho \Theta_{S}-\frac{m}{4 \hbar^{2}} \rho \Theta_{V}\right)^{-1} .
$$

By using $m^{*}$ instead of $m$ in Eq. (5), new EOS's for symmetric and pure neutron matter are obtained. In the case of cutoff regularization, they are evaluated by multiplying Eqs. (22), (23), and (27), (28) by Eqs. (36) and (37), respectively. In the case of dimensional regularization, they are obtained 


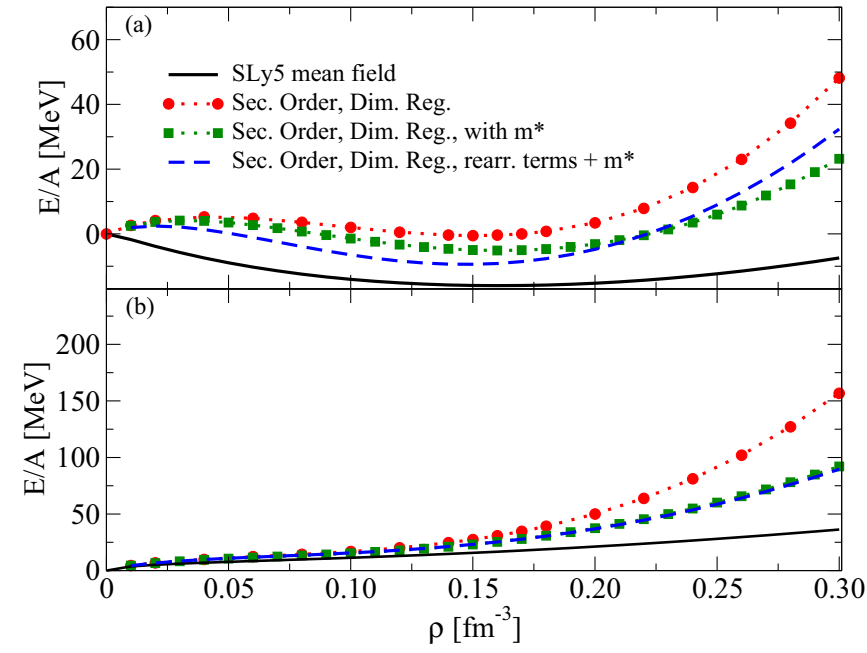

FIG. 26. Second-order EOS's for symmetric (a) and pure neutron matter (b) compared with the SLy5-mean-field EOS's, calculated with the SLy5 parameters for the case where $m^{*}=m$ and rearrangement terms are neglected (red circles and dotted line), for the case where a mean-field effective mass is used and rearrangement terms are neglected (gree squares and dotted line), and for the case where a mean-field effective mass is used and rearrangement terms are included (blue dashed line).

by multiplying Eqs. (34) and (35) by Eqs. (36) and (37), respectively. The corresponding curves evaluated with the SLy5 parameters show a density-dependent rescaling effect, as obviously expected. This may be observed in the illustrative case of cutoff regularization: Figures 23 and 24 describe the same quantities as Figs. 1 and 7 , but with $m^{*} \neq m$.

Fits of parameters may be done for symmetric and pure neutron matter and we provide here the illustrative results obtained in the case of the cutoff regularization. We show in Fig. 25 the curves corresponding to a global fit done on the benchmark SLy5 EOS's by including simultaneously symmetric and pure neutron matter. The obtained parameters are listed in Table VI. The incompressibility modulus ranges from 202 to $238 \mathrm{MeV}$, according to the different cutoff values. The quality of the fit is globally very good with a maximum average deviation from the reference curve of $\sim 3 \%$. We mention that, in the case of dimensional regularization, we found that the inclusion of an effective mass is not sufficient to improve the quality of the fit of the second-order EOS for symmetric matter, which remains similar to that shown in Fig. 18.

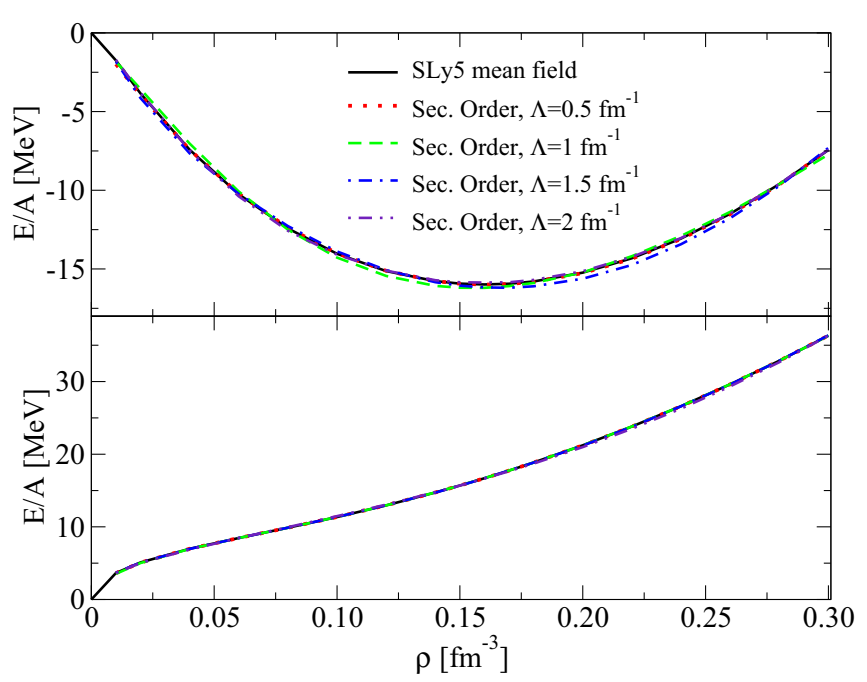

FIG. 27. Second-order EOS's for symmetric (a) and pure neutron matter (b) adjusted on the SLy5-mean-field EOS's, with an effective mass equal to the mean-field effective mass and with the inclusion of the rearrangement terms, for different values of the cutoff.

We discuss now the rearrangement terms. As already anticipated in Sec. I, Ref. [18] pointed out that the square of the interaction entering in the computation of the second-order energy correction coincides with the square of the RPA $B$ matrix, which means that the rearrangement terms have to be computed by using the second derivative of the HartreeFock energy functional, as done in RPA. By following such procedure and using the Landau parameters computed with the Skyrme force for symmetric and pure neutron matter [26], the combinations of parameters containing the $t_{3}$ part in Eqs. (24) and (29) may be replaced by

$$
\begin{aligned}
\widetilde{T^{R}}{ }_{03}^{2}= & {\left[t_{0}\left(1-x_{0}\right)+\frac{1}{6} t_{3}\left(1-x_{3}\right) \rho^{\alpha}+\frac{1}{32} t_{3} \rho^{\alpha} \alpha(3+\alpha)\right]^{2} } \\
& +\left[t_{0}\left(1+x_{0}\right)+\frac{1}{6} t_{3}\left(1+x_{3}\right) \rho^{\alpha}+\frac{1}{32} t_{3} \rho^{\alpha} \alpha(3+\alpha)\right]^{2}, \\
\widetilde{T^{R}}{ }_{03}{\widetilde{T R^{R}}}_{1}= & \frac{t_{1}}{2}\left[\left[t_{0}\left(1-x_{0}\right)+\frac{1}{6} t_{3}\left(1-x_{3}\right) \rho^{\alpha}+\frac{1}{32} t_{3} \rho^{\alpha} \alpha(3+\alpha)\right]\right. \\
& \left.\times\left(1-x_{1}\right)\right]+\frac{t_{1}}{2}\left[\left[t_{0}\left(1+x_{0}\right)+\frac{1}{6} t_{3}\left(1+x_{3}\right) \rho^{\alpha}\right.\right. \\
& \left.\left.+\frac{1}{32} t_{3} \rho^{\alpha} \alpha(3+\alpha)\right]\left(1+x_{1}\right)\right],
\end{aligned}
$$

\begin{tabular}{|c|c|c|c|c|c|c|c|c|c|c|}
\hline & $\begin{array}{c}t_{0} \\
\left(\mathrm{MeV} \mathrm{fm}^{3}\right)\end{array}$ & $\begin{array}{c}t_{1} \\
\left(\mathrm{MeV} \mathrm{fm}^{5}\right)\end{array}$ & $\begin{array}{c}t_{2} \\
\left(\mathrm{MeV} \mathrm{fm}^{5}\right)\end{array}$ & $\begin{array}{c}t_{3} \\
\left(\mathrm{MeV} \mathrm{fm}^{3+3 \alpha}\right)\end{array}$ & $x_{0}$ & $x_{1}$ & $x_{2}$ & $x_{3}$ & $\alpha$ & $\chi^{2}$ \\
\hline $\begin{array}{l}\text { SLy5 } \\
\Lambda\left(\mathrm{fm}^{-1}\right)\end{array}$ & -2484.88 & 483.13 & -549.40 & 13736.0 & 0.778 & -0.328 & -1.0 & 1.267 & 0.16667 & \\
\hline 0.5 & -2226.39 & 833.57 & -1054.76 & 12615.02 & 0.7243 & -0.3572 & -0.8483 & 1.2719 & 0.2020 & 0.03 \\
\hline 1.0 & -9.43 & 1004.59 & -3683.79 & -10057.96 & -21.7819 & 3.1346 & -1.2868 & -0.2921 & 0.8324 & 3.48 \\
\hline 1.5 & 520.74 & 664.84 & -2226.01 & -8415.55 & 1.7992 & -1.6278 & -1.3517 & -6.5449 & 0.6387 & 2.15 \\
\hline
\end{tabular}

TABLE VII. Same as in Table VI, with also the inclusion of rearrangement terms. 


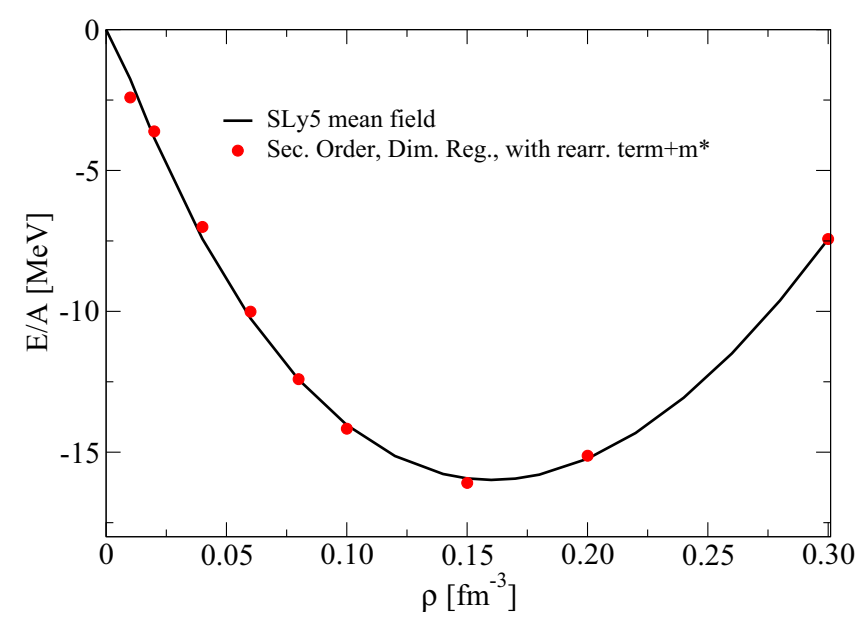

FIG. 28. Same as in Fig. 18, but with an effective mass equal to the mean-field effective mass and with the inclusion of the rearrangement terms.

$$
\begin{aligned}
T_{03}^{R}= & t_{0}\left(1-x_{0}\right)+\frac{1}{6} t_{3}\left(1-x_{3}\right) \rho^{\alpha} \\
& +\frac{1}{48} t_{3} \rho^{\alpha} \alpha(\alpha+3)\left(1-x_{3}\right),
\end{aligned}
$$

where $R$ indicates the inclusion of the rearrangement terms.

Figure 26 shows, as an illustration, how the dimensionalregularized second-order EOS's (computed with the SLy5 parameters) are modified by the inclusion of the rearrangement terms. One observes that, for the case of neutron matter, the inclusion of rearrangement terms in the EOS has a very weak effect compared to the much more important effect coming from the inclusion of the effective mass. On the other side, for the case of symmetric matter, rearrangement terms modify the curve. Such modification, with respect to the case where rearrangement terms were omitted, allows us to obtain a much better refitted dimensional-regularized EOS for symmetric matter, as will be shown below.

For the case of cutoff regularization, we present in Fig. 27 the curves obtained with a global fit including symmetric and neutron matter (the corresponding parameters and $\chi^{2}$ can be found in Table VII). The incompressibility modulus ranges from 200 to $250 \mathrm{MeV}$, according to the different cutoff values, and the quality of the fit is very good as indicated by the $\chi^{2}$ values.

The same global fit does not provide any good results for the case of dimensional regularization. We have then performed separately the two fits for symmetric and neutron matter (Figs. 28 and 29 and Tables VIII and IX). The incompressibility modulus is equal to $250 \mathrm{MeV}$. The quality of the fit is now less

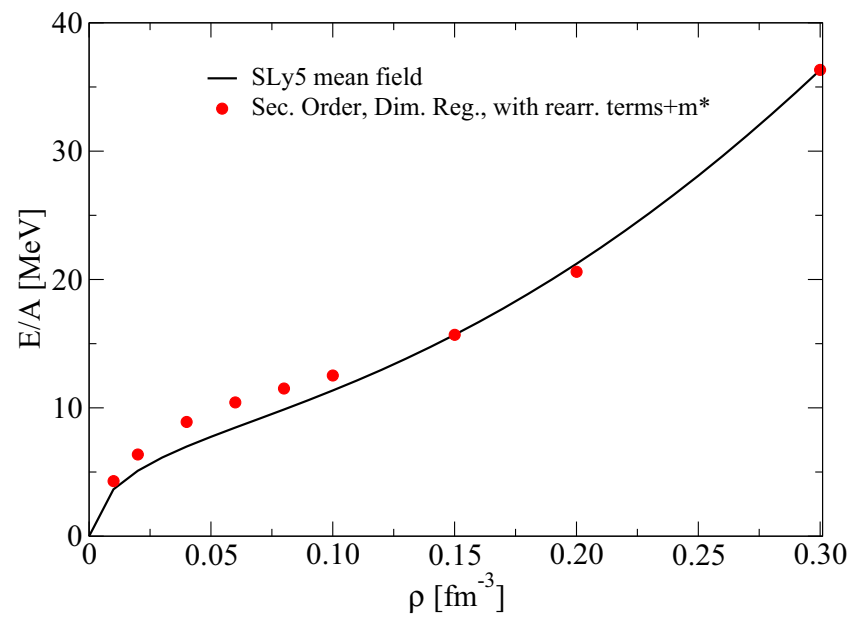

FIG. 29. Same as in Fig. 28, but for neutron matter.

good but still reasonably good in the case of symmetric matter (average deviation of 13\%) and acceptable for neutron matter (average deviation of $21 \%$ ).

We see here the importance of including the densitydependent part of the Skyrme force. The inclusion of this part without the rearrangement terms allowed us in the previous section to shift the equilibrium point of symmetric matter to the correct one (compared to the EOS obtained in Ref. [13] where the $t_{3}$ part of the Skyrme force was totally neglected). The inclusion of the rearrangement terms allows us now to correctly describe the EOS of symmetric matter also in the other density regions and to have a reasonable value for the incompressibility modulus.

\section{CONCLUSIONS}

We have presented a study devoted to the computation of the second-order correction in the EOS of symmetric, neutron, and asymmetric matter, with the use of the Skyrme effective interaction. Owing to the zero range of such force, the secondorder contribution to the nuclear-matter EOS diverges and a momentum cutoff must be used to regularize the divergent integrals. The divergence is linear in the momentum cutoff in the simplified $t_{0}-t_{3}$ model [8] and goes like the fifth power of the cutoff in the case where the velocity-dependent terms of the Skyrme interaction are also included. For simplicity, we have omitted the spin-orbit and tensor terms in the used expression of the Skyrme interaction. In addition to the occurrence of an ultraviolet divergence, second-order calculations performed with such an effective interaction present also a well-known risk of double counting, because the parameters of the force are

TABLE VIII. Parameter set obtained for symmetric matter with the fit of the dimensional-regularized second-order EOS compared with

\begin{tabular}{|c|c|c|c|c|c|c|c|c|c|c|}
\hline & $\begin{array}{c}t_{0} \\
\left(\mathrm{MeV} \mathrm{fm}^{3}\right)\end{array}$ & $\begin{array}{c}t_{1} \\
\left(\mathrm{MeV} \mathrm{fm}^{5}\right)\end{array}$ & $\begin{array}{c}t_{2} \\
\left(\mathrm{MeV} \mathrm{fm}^{5}\right)\end{array}$ & $\begin{array}{c}t_{3} \\
\left(\mathrm{MeV} \mathrm{fm}{ }^{3+3 \alpha}\right)\end{array}$ & $x_{0}$ & $x_{1}$ & $x_{2}$ & $x_{3}$ & $\alpha$ & $\chi^{2}$ \\
\hline SLy5 & $\begin{array}{l}-2484.88 \\
-1425.43\end{array}$ & $\begin{array}{c}483.13 \\
-16732.70\end{array}$ & $\begin{array}{c}-549.40 \\
1345.58\end{array}$ & $\begin{array}{r}13763.0 \\
373005.96\end{array}$ & $\begin{array}{c}0.778 \\
-0.0279\end{array}$ & $\begin{array}{l}-0.328 \\
-0.1885\end{array}$ & $\begin{array}{c}-1.0 \\
-5.2091\end{array}$ & $\begin{array}{c}1.267 \\
-0.2014\end{array}$ & $\begin{array}{l}0.16667 \\
0.5796\end{array}$ & 184 \\
\hline
\end{tabular}
the original set SLy5. The mean-field effective mass and rearrangement terms are included. The $\chi^{2}$ value is shown in the last column. 
TABLE IX. Same as in Table VIII but for neutron matter.

\begin{tabular}{cccccccccc}
\hline \hline & $\begin{array}{c}t_{0} \\
\left(\mathrm{MeV} \mathrm{fm}^{3}\right)\end{array}$ & $\begin{array}{c}t_{1} \\
\left(\mathrm{MeV} \mathrm{fm}^{5}\right)\end{array}$ & $\begin{array}{c}t_{2} \\
\left(\mathrm{MeV} \mathrm{fm}^{5}\right)\end{array}$ & $\begin{array}{c}t_{3} \\
\left(\mathrm{MeV} \mathrm{fm}^{3+3 \alpha}\right)\end{array}$ & $x_{0}$ & $x_{1}$ & $x_{2}$ & $x_{3}$ \\
\hline SLy5 & -2484.88 & 483.13 & -549.40 & 13763.0 & 0.778 & -0.328 & -1.0 & 1.267 & 0.16667 \\
& 1591.32 & -837.21 & -1498.53 & 2582.80 & 1.5534 & 1.7740 & -0.9306 & 7.2733 & 1.3874 \\
\hline \hline
\end{tabular}

adjusted to reproduce observables with leading-order (meanfield) calculations. This implies that the parameters already contain in an implicit way some correlations. The cancellation of such double counting is then required.

We have treated the ultraviolet divergence appearing at second order in the EOS of nuclear matter by using cutoff and dimensional regularizations, as was done in Refs. [9,10], respectively: in the first case, the full second-order correction is calculated with all the cutoff-dependent terms. The analytical derivation of all the terms is presented for symmetric and neutron matter. Results obtained numerically with a Monte Carlo integration for symmetric, neutron, and asymmetric matter are discussed. For each value of the introduced momentum cutoff, a new set of parameters is obtained by adjusting the secondorder EOS to the chosen benchmark SLy5-mean-field EOS. This procedure eliminates both double-counting problems and divergences. In the case of dimensional regularization, only the finite part of the EOS is kept. In such a case, only double-counting problems arise and they are removed also, this time by an adjustment of the parameters. Unique sets of parameters are produced for each type of EOS (no cutoff). The objective of this work is to present in a complete and detailed form revised results and figures with respect to those illustrated in Refs. $[9,10]$. We have realized recently that those results are incomplete in some aspects concerning the analytical derivation of specific second-order contributions. In addition, we have evaluated the effects associated to the rearrangement terms entering in second-order calculations and related to the density dependence of the interaction. Sets of parameters adjusted for symmetric and neutron matter, taking into account an effective mass evaluated at the mean-field level, and including also the proper rearrangement terms, are provided. Such sets may be considered a very reasonable starting point within our general objective; that is, the construction of generalized effective interactions that are specially designed to be used in a beyond-mean-field scheme: parameters are adjusted at the same level of the performed calculations (no double counting) and produce results which are independent of the chosen energy or momentum cutoff. Such regularized interactions will open the possibility of performing robust beyond-mean-field applications to finite nuclei.

\section{ACKNOWLEDGMENT}

G. C. acknowledges discussions with N. Kaiser and M.G. aknowledges discussions with A. Pastore.

\section{APPENDIX: FACTORS IN FRONT OF THE INTEGRALS}

The second-order correction written in the proton $(p)$ and neutron $(n)$ basis is the sum of $n n, p p$, and $n p$ contributions, that is,

$$
\begin{aligned}
\Delta E^{(2)}= & \frac{1}{2} \sum_{i j}\langle i j|V G V| i j\rangle_{p p}+\frac{1}{2} \sum_{i j}\langle i j|V G V| i j\rangle_{n n} \\
& +\sum_{i j}\langle i j|V G V| i j\rangle_{n p} .
\end{aligned}
$$

Here $i$ and $j$ are the labels of the two particles. Note that $\sum_{i j}=\frac{\Omega^{3}}{(2 \pi)^{9}} \int d^{3} \mathbf{k}_{1} \int d^{3} \mathbf{k}_{2} \int d^{3} \mathbf{q}$, and $V=v / \Omega$. For the $n p$ case, we associate $i$ to $n$ and $j$ to $p$. The factor 2 in the $n p$ part with respect to $n n$ and $p p$ parts accounts for the symmetric $p n$ contribution.

When evaluating the matrix element, the exchange term is included by inserting $\left(1-P_{x} P_{\sigma} P_{\tau}\right)$ from the left, where $P_{x}, P_{\sigma}, P_{\tau}$ are the space, spin, and isospin exchange operators, respectively. The exchange contribution is thus equal to the direct one in the $n n$ and $p p$ channels in the cases of even-even and odd-odd mixing of the interaction. In the cases of even-odd mixing of the interaction, one has $1-P_{x} P_{\sigma} P_{\tau}=1+P_{\sigma} P_{\tau}$ for the $n n$ and $p p$ channels ( $P_{x}$ provides a minus sign). The exchange term is always equal to zero in the $n p$ channel.

\section{1. $t_{0}^{2}\left(t_{3}^{2}\right)$ matrix element}

We start with the square of the term $v=t_{0}\left(1+x_{0} P_{\sigma}\right)$ (analogous expressions may be written for the square of the $t_{3}$ term).

(a) $n n$ channel. In this case, $T=1$. The mixing of the interaction is even-even $(l=0)$ and this leads to $S=0 . P_{\sigma}$ acting on the spin singlet state provides a minus sign. One has

$$
\begin{aligned}
\frac{1}{2} \sum_{i j}\langle i j|V G V| i j\rangle_{n n} \\
=\frac{t_{0}^{2} \Omega}{(2 \pi)^{9}} \int d^{3} \mathbf{k}_{1} \int d^{3} \mathbf{k}_{2} \int d^{3} \mathbf{q} G \\
\quad \times\left[\sum_{S M_{S}}\left\langle X_{M_{S}}^{S}\left|\left(1+2 x_{0} P_{\sigma}+x_{0}^{2}\right)\right| X_{M_{S}}^{S}\right\rangle\right],
\end{aligned}
$$

where $M_{S}$ is the spin projection. By applying the spin exchange operator and by performing the sum (there is only one term: $S=0 ; M_{S}=0$ ) one has

$$
\begin{aligned}
& \frac{1}{2} \sum_{i j}\langle i j|V G V| i j\rangle_{n n} \\
& \quad=\frac{t_{0}^{2} \Omega}{(2 \pi)^{9}}\left[1-2 x_{0}+x_{0}^{2}\right] \int d^{3} \mathbf{k}_{1} \int d^{3} \mathbf{k}_{2} \int d^{3} \mathbf{q} G
\end{aligned}
$$


We introduce the quantities

$$
\begin{aligned}
\overline{\mathbf{k}}_{1}=\frac{\mathbf{k}_{1}}{k_{N}}, & \overline{\mathbf{k}}_{2}=\frac{\mathbf{k}_{2}}{k_{N}}, \quad \overline{\mathbf{q}}=\frac{\mathbf{q}}{k_{N}}, \\
\overline{\mathbf{k}}=\frac{\mathbf{k}}{k_{N}}, & \overline{\mathbf{k}^{\prime}}=\frac{\mathbf{k}^{\prime}}{k_{N}}, \\
\bar{\Lambda} & =\frac{\Lambda}{k_{N}}, \quad \bar{G}=G k_{N}^{2} .
\end{aligned}
$$

Then

$$
\frac{1}{2} \sum_{i j}\langle i j|V G V| i j\rangle_{n n}=\frac{t_{0}^{2} \Omega}{(2 \pi)^{9}}\left[1-2 x_{0}+x_{0}^{2}\right] k_{F_{N}}^{7} \int_{0}^{1} d^{3} \overline{\mathbf{k}}_{1} \int_{0}^{1} d^{3} \overline{\mathbf{k}}_{2} \int_{0}^{\bar{\Lambda}} d^{3} \overline{\mathbf{q}} \bar{G} .
$$

(b) $p p$ channel:

The $p p$ contribution is the same as the $n n$ contribution, with $k_{F_{N}} \rightarrow k_{F_{P}}$ in Eq. (A5).

(c) $n p$ channel (in the $n p$ channel, the operator $P_{\sigma}$ provides a plus sign when applied to the spin triplet states and a minus sign when applied to the spin singlet state).

We introduce the quantity

$$
a=\frac{k_{F_{P}}}{k_{F_{N}}}=\left(\frac{1-\delta}{1+\delta}\right)^{1 / 3}
$$

Then

$$
\begin{aligned}
\sum_{i j}\langle i j|V G V| i j\rangle_{n p} & =\frac{t_{0}^{2} \Omega}{(2 \pi)^{9}} k_{F_{N}}^{7} \int_{0}^{1} d^{3} \overline{\mathbf{k}}_{1} \int_{0}^{a} d^{3} \overline{\mathbf{k}}_{2} \int_{0}^{\bar{\Lambda}} d^{3} \overline{\mathbf{q}} \bar{G}\left[\sum_{S M_{S}}\left\langle X_{M_{S}}^{S}\left|\left(1+2 x_{0} P_{\sigma}+x_{0}^{2}\right)\right| X_{M_{S}}^{S}\right\rangle\right] \\
& =4 \frac{t_{0}^{2} \Omega}{(2 \pi)^{9}} k_{F_{N}}^{7}\left(1+x_{0}+x_{0}^{2}\right) \int_{0}^{1} d^{3} \overline{\mathbf{k}}_{1} \int_{0}^{a} d^{3} \overline{\mathbf{k}}_{2} \int_{0}^{\bar{\Lambda}} d^{3} \overline{\mathbf{q}} \bar{G}
\end{aligned}
$$

\section{2. $t_{0}\left(t_{3}\right) t_{2}$ part}

This contribution is equal to zero in the case of symmetric and neutron matter, because it mixes even and odd terms of the interaction. One has

$$
v G v=t_{0}\left(1+x_{0} P_{\sigma}\right) t_{2}\left(1+x_{2} P_{\sigma}\right) \mathbf{k}^{\prime} \cdot \mathbf{k} G
$$

By including the direct and exchange terms,

$$
\langle i j|v G v| i j\rangle=\mathbf{k}^{\prime} \cdot \mathbf{k} G\left\langle i j\left|t_{0} t_{2}\left(1+x_{2} P_{\sigma}+x_{0} P_{\sigma}+x_{0} x_{2}\right)\left(1+P_{\sigma} P_{\tau}\right)\right| i j\right\rangle .
$$

(a) $n n$ channel:

In this case, both particles are neutrons and the antisymmetrization condition must be imposed. Therefore, as in Sec. II A, the odd-even mixing of the interaction is not allowed. Thus,

$$
\sum_{i j}\langle i j|V G V| i j\rangle_{n n}=0
$$

(b) $p p$ channel:

The $p p$ part is the same as the $n n$ contribution and thus equal to zero.

(c) $n p$ channel:

Now the two particles are not identical if their densities are different (different Fermi momenta). We have the following nonvanishing term:

$$
\begin{aligned}
\sum_{i j}\langle i j|V G V| i j\rangle_{n p} & =\frac{t_{0} t_{2} \Omega}{(2 \pi)^{9}} k_{F_{N}}^{9} \int_{0}^{1} d^{3} \overline{\mathbf{k}}_{1} \int_{0}^{a} d^{3} \overline{\mathbf{k}}_{2} \int_{0}^{\bar{\Lambda}} d^{3} \overline{\mathbf{q}} \overline{\mathbf{k}}^{\prime} \cdot \overline{\mathbf{k}} \bar{G}\left[\sum_{S M_{S}}\left\langle X_{M_{S}}^{S}\left|1+\left(x_{0}+x_{2}\right) P_{\sigma}+x_{0} x_{2}\right| X_{M_{S}}^{S}\right\rangle\right] \\
& =2 \frac{t_{0} t_{2} \Omega}{(2 \pi)^{9}} k_{F_{N}}^{9}\left(2+x_{0}+x_{2}+2 x_{0} x_{2}\right) \int_{0}^{1} d^{3} \overline{\mathbf{k}}_{1} \int_{0}^{a} d^{3} \overline{\mathbf{k}}_{2} \int_{0}^{\bar{\Lambda}} d^{3} \overline{\mathbf{q}} \overline{\mathbf{k}}^{\prime} \cdot \overline{\mathbf{k}} \bar{G} .
\end{aligned}
$$

Note that if protons and neutrons have the same density, then they can be considered as identical particles. This is reflected in the above equation: by setting $a=1$, the integral leads to zero, as for the $n n$ and $p p$ cases. 
All the other matrix elements can be evaluated in the same way as above. We list the results of the other terms below.

\section{3. $t_{0}\left(t_{3}\right) t_{1}$ part}

(a) $n n$ channel:

$$
\frac{1}{2} \sum_{i j}\langle i j|V G V| i j\rangle_{n n}=\frac{1}{2} \frac{t_{0} t_{1} \Omega}{(2 \pi)^{9}} k_{F_{N}}^{9}\left(1-x_{0}-x_{1}+x_{0} x_{1}\right) \int_{0}^{1} d^{3} \overline{\mathbf{k}}_{1} \int_{0}^{1} d^{3} \overline{\mathbf{k}}_{2} \int_{0}^{\bar{\Lambda}} d^{3} \overline{\mathbf{q}}\left(\overline{\mathbf{k}}^{2}+\overline{\mathbf{k}}^{2}\right) \bar{G}
$$

(b) $p p$ channel:

The $p p$ part is the same as the $n n$ part, by replacing $k_{F_{N}} \rightarrow k_{F_{P}}$ in Eq. (A12).

(c) $n p$ channel:

$$
\sum_{i j}\langle i j|V G V| i j\rangle_{n p}=\frac{t_{0} t_{1} \Omega}{(2 \pi)^{9}} k_{F_{N}}^{9}\left(2+x_{0}+x_{1}+2 x_{0} x_{1}\right) \int_{0}^{1} d^{3} \overline{\mathbf{k}}_{1} \int_{0}^{a} d^{3} \overline{\mathbf{k}}_{2} \int_{0}^{\bar{\Lambda}} d^{3} \overline{\mathbf{q}}\left(\overline{\mathbf{k}}^{2}+\overline{\mathbf{k}}^{2}\right) \bar{G} .
$$

\section{4. $t_{1} t_{2}$ part}

(a) $n n$ channel. It is analogous to the $n n$ part of the $t_{0} t_{2}$ part (even-odd mixing):

$$
\sum_{i j}\langle i j|V G V| i j\rangle_{n n}=0 .
$$

(b) $p p$ channel:

The $p p$ part is the same as the $n n$ part and is equal to zero.

(c) $n p$ channel:

$$
\sum_{i j}\langle i j|V G V| i j\rangle_{n p} \quad=\frac{t_{1} t_{2} \Omega}{(2 \pi)^{9}} k_{F_{N}}^{11}\left(2+x_{1}+x_{2}+2 x_{1} x_{2}\right) \int_{0}^{1} d^{3} \overline{\mathbf{k}}_{1} \int_{0}^{a} d^{3} \overline{\mathbf{k}}_{2} \int_{0}^{\bar{\Lambda}} d^{3} \overline{\mathbf{q}}\left(\overline{\mathbf{k}}^{\prime 2}+\overline{\mathbf{k}}^{2}\right) \overline{\mathbf{k}}^{\prime} \cdot \overline{\mathbf{k} G} .
$$

\section{5. $t_{1}^{2}$ part}

(a) $n n$ channel:

$$
\frac{1}{2} \sum_{i j}\langle i j|V G V| i j\rangle_{n n}=\frac{1}{4} \frac{t_{1}^{2} \Omega}{(2 \pi)^{9}}\left[1-2 x_{1}+x_{1}^{2}\right] k_{F_{N}}^{11} \int_{0}^{1} d^{3} \overline{\mathbf{k}}_{1} \int_{0}^{1} d^{3} \overline{\mathbf{k}}_{2} \int_{0}^{\bar{\Lambda}} d^{3} \overline{\mathbf{q}}\left(\overline{\mathbf{k}}^{2}+\overline{\mathbf{k}}^{2}\right)^{2} \bar{G},
$$

(b) $p p$ channel:

The $p p$ part is the same as the $n n$ part, with $k_{F_{N}} \rightarrow k_{F_{P}}$.

(c) $n p$ channel:

$$
\sum_{i j}\langle i j|V G V| i j\rangle_{n p}=\frac{t_{1}^{2} \Omega}{(2 \pi)^{9}} k_{F_{N}}^{11}\left(1+x_{1}+x_{1}^{2}\right) \int_{0}^{1} d^{3} \overline{\mathbf{k}}_{1} \int_{0}^{a} d^{3} \overline{\mathbf{k}}_{2} \int_{0}^{\bar{\Lambda}} d^{3} \overline{\mathbf{q}}\left(\overline{\mathbf{k}}^{2}+\overline{\mathbf{k}}^{2}\right)^{2} \bar{G} .
$$

\section{6. $t_{2}^{2}$ part}

(a) $n n$ channel. In this case, $T=1$. The mixing of the interaction is odd-odd $(l=1)$ and this leads to $S=1$. $P_{\sigma}$ provides a plus sign for each of the triplet states:

$$
\frac{1}{2} \sum_{i j}\langle i j|V G V| i j\rangle_{n n}=3 \frac{t_{2}^{2} \Omega}{(2 \pi)^{9}}\left[1+2 x_{2}+x_{2}^{2}\right] k_{F_{N}}^{11} \int_{0}^{1} d^{3} \overline{\mathbf{k}}_{1} \int_{0}^{1} d^{3} \overline{\mathbf{k}}_{2} \int_{0}^{\bar{\Lambda}} d^{3} \overline{\mathbf{q}}\left(\overline{\mathbf{k}}^{\prime} \cdot \overline{\mathbf{k}}\right)^{2} \bar{G},
$$

(b) $p p$ channel:

The $p p$ part is the same as the $n n$ part, with $k_{F_{N}} \rightarrow k_{F_{P}}$.

(c) $n p$ channel:

$$
\sum_{i j}\langle i j|V G V| i j\rangle_{n p}=4 \frac{t_{2}^{2} \Omega}{(2 \pi)^{9}} k_{F_{N}}^{11}\left(1+x_{2}+x_{2}^{2}\right) \int_{0}^{1} d^{3} \overline{\mathbf{k}}_{1} \int_{0}^{a} d^{3} \overline{\mathbf{k}}_{2} \int_{0}^{\bar{\Lambda}} d^{3} \overline{\mathbf{q}}\left(\overline{\mathbf{k}}^{\prime} \cdot \overline{\mathbf{k}}\right)^{2} \bar{G} .
$$


[1] M. Bender, P.-H. Heenen, and P.-G. Reinhard, Rev. Mod. Phys. 75, 121 (2003).

[2] M. Kortelainen, J. McDonnell, W. Nazarewicz, P.-G. Reinhard, J. Sarich, N. Schunck, M. V. Stoitsov, and S. M. Wild, Phys. Rev. C 85, 024304 (2012).

[3] A. L. Fetter and J. D. Walecka, Quantum Theory of Many particle Systems (Dover, Mineola, NY, 2003).

[4] T. H. R. Skyrme, Philos. Mag. 1, 1043 (1956); Nucl. Phys. 9, 615 (1959).

[5] D. Vautherin and D. M. Brink, Phys. Rev. C 5, 626 (1972).

[6] J. Dechargé and D. Gogny, Phys. Rev. C 21, 1568 (1980).

[7] J. F. Berger, M. Girod, and D. Gogny, Comput. Phys. Commun. 63, 365 (1991).

[8] K. Moghrabi, M. Grasso, G. Colò, and N. Van Giai, Phys. Rev. Lett. 105, 262501 (2010).

[9] K. Moghrabi, M. Grasso, X. Roca-Maza, and G. Colò, Phys. Rev. C 85, 044323 (2012).

[10] K. Moghrabi and M. Grasso, Phys. Rev. C 86, 044319 (2012).

[11] M. Brenna, G. Colò, and X. Roca-Maza, Phys. Rev. C 90, 044316 (2014).

[12] M. Waroquier, J. Ryckebush, J. Moreau, K. Heyde, N. Blasi, S. Y. van der Werf, and G. Wenes, Phys. Rep. 148, 249 (1987).
[13] N. Kaiser, J. Phys. G: Nucl. and Part. Phys. 42, 095111 (2015).

[14] S. Stringari and D. M. Brink, Nucl. Phys. A 304, 307 (1978).

[15] D. Lacroix, T. Duguet, and M. Bender, Phys. Rev. C 79, 044318 (2009).

[16] N. M. Hugenholtz and L. Van Howe, Physica 24, 363 (1958).

[17] D. Gambacurta, M. Grasso, and F. Catara, J. Phys. G Nucl. Part. Phys. 38, 035103 (2011).

[18] B. G. Carlsson, J. Toivanen, and U. von Barth, Phys. Rev. C 87, 054303 (2013).

[19] P. Ring and P. Schuck, The Nuclear Many-Body Problem (Springer, New York, 1980).

[20] S. K. Bogner, T. T. S. Kuo, A. Schwenk, D. R. Entem, and R. Machleidt, Phys. Lett. B 576, 265 (2003).

[21] K. T. R. Davies and M. Baranger, Nucl. Phys. A 120, 254 (1968).

[22] E. Chabanat, P. Bonche, P. Haensel, J. Meyer, and R. Schaeffer, Nucl. Phys. A 627, 710 (1997); 635, 231 (1998); 643, 441 (1998).

[23] G. 't Hooft and M. J. G. Veltman, Nucl. Phys. B 44, 189 (1972).

[24] G. 't Hooft, Nucl. Phys. B 61, 455 (1973).

[25] C. G. Bollini and J. J. Giambiagi, Nuovo Cimento B 12, 20 (1972).

[26] A. Pastore, D. Davesne, and J. Navarro, Phys. Rep. 563, 1 (2015). 